\title{
Bulletin of the School of Oriental and African Studies
}

http://journals.cambridge.org/BSO

Additional services for Bulletin of the School of Oriental and African Studies:

Email alerts: Click here

Subscriptions: $\underline{\text { Click here }}$

Commercial reprints: $\underline{\text { Click here }}$

Terms of use : $\underline{\text { Click here }}$

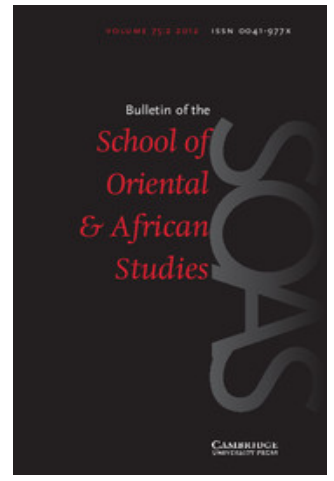

\section{Verbal Phrases in Lhasa Tibetan-III}

R. K. Sprigg

Bulletin of the School of Oriental and African Studies / Volume 16 / Issue 03 / October 1954, pp 566 - 591

DOI: 10.1017/S0041977X00086869, Published online: 24 December 2009

Link to this article: http://journals.cambridge.org/abstract_S0041977X00086869

How to cite this article:

R. K. Sprigg (1954). Verbal Phrases in Lhasa Tibetan-III. Bulletin of the School of Oriental and African Studies, 16, pp 566-591 doi:10.1017/S0041977X00086869

Request Permissions : $\underline{\text { Click here }}$ 


\title{
VERBAL PHRASES IN LHASA TIBETAN-III
}

\author{
By R. K. SprigG
}

əW : EXPONENTS OF ' $ə$ '; PROSODIC AND PHONEMATIC SYSTEMS

There is one important respect in which $ə \mathrm{~W}$ differs from $\mathrm{yW}$ and $\mathrm{wW}$. For these last it was sufficient to state exponents of ' $y$ ' and ' $w$ '; the exponents of ' $a$ ' however can be stated only by introducing a further prosodic system, of the initial syllable, $y / w$, i.e. ' $y$ neutral words' ( $y ə W$ ) and ' $w$ neutral words' (woW). The prosodic and phonematic systems of yoW resemble those stated above for the initial syllable of $y W$, and those of waW resemble the systems stated for the initial syllable of $\mathrm{wW}$, i.e.

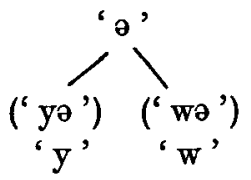

The two-term $y / w$ system ( $y$ o/wo) is stated for the initial syllable of the (e) word, and is not to be confused with the two-term prosodic system of the syllable initial $(y / w I)$. The exponents of $y$ and $w$ in the initial syllable (CVC) of $ə \mathrm{~W}$ comprise :-
A. $-\mathrm{V}-:$ : frontness + spreading
$\stackrel{w}{c}$
frontness - spreading,
backness ;
B. C- :
velarity,
absence of consonant articulation

e.g.

A. $-\mathrm{V}-\mathrm{y}:$

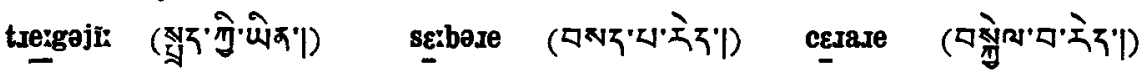
$\mathrm{w}$ :

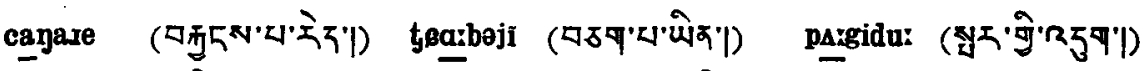

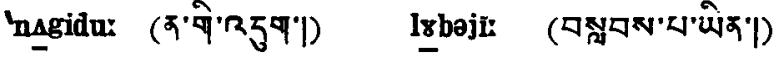

B. C- :

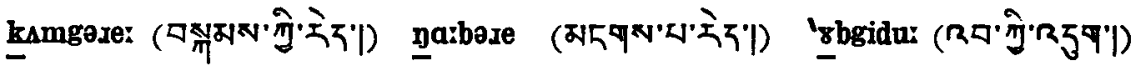
C. $-\mathrm{C}$ :

The phonetic exponents need to be stated with reference to the c/o system :-$c$ w: $\quad$ labiality

cW : 'lrbgidu:

'damgidu:

oW : teagbeji

'eanare

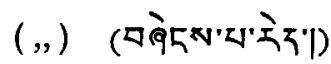

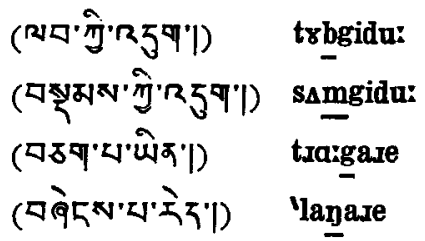
velarity, e.g. (श्रेषN.गे'Rुुप।)

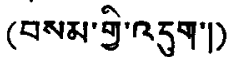

(fS) (ब्रेष'च'亡ेर')

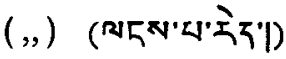


Where the $-\mathrm{C}$ term is $-\mathrm{T}$, there are as exponents of $\mathrm{y} / \mathrm{w}:-$

$y$ : long duration of vowel ; $w$ : short duration of vowel (cf. also $y W$, wW), e.g.

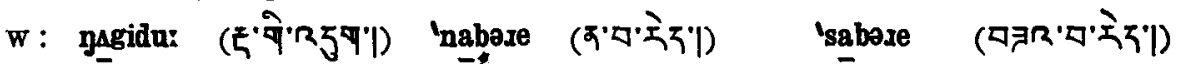

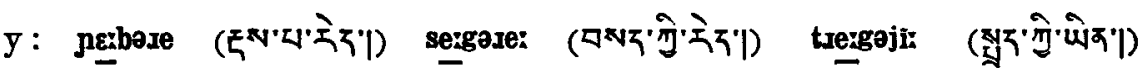

I. EXPONENTS OF ' $\theta$ '

Exponents ${ }^{x}$ of ' $\partial$ ' may be stated for the initial and medial syllables; exponents of non-' $w$ ' may be stated for the final syllable also.

The exponents of $\partial$ are as follows :-

\section{A. Initial Syllable $(C V C)$}

(1) $-\mathrm{V}$ - is characterized by

(a) backness - rounding (cf. $\mathrm{wW}$; backness + rounding)

(b) frontness - spreading (, $\mathrm{yW}$; frontness + spreading), e.g. :-

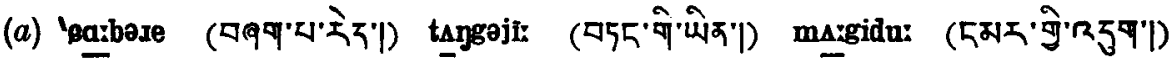 'Irbgidu: (लव.गुरेखुष'|)

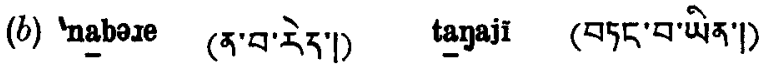

(2) C- (wəW)

(a) absence of lip-spreading or lip-rounding (cf. $\mathrm{yW} / \mathrm{wW}$ ),

(b) absence of consonant articulation, e.g. :-
(a) traibore

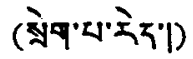
layare
( अॅ5
(b) 'rbese
(Rロン・主们)

\section{B. Medial Syllable $\left(C_{1} V_{1}\right)$}

In $\mathrm{cW}-\mathrm{V}_{1}$ may be characterized by centralization, e.g. :-

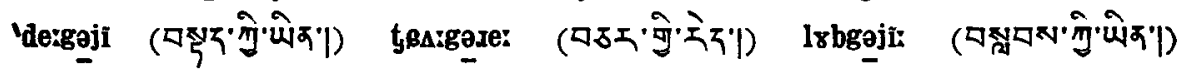

C. Final Syllable $\left(C_{2} V_{2}\right)$

Exponents may be stated for ' non- $w$ ', but not for ' $\rho$ '.

The stating of the exponents of ' $a$ ' completes that of the three terms $w / y / \partial$. It is, however, also possible to state exponents for two of these terms as against the third, e.g. of (A) 'non-y', (B) 'non-w', and of (C) ' $w$ ' and 'wo' as against ' $y$ ' and ' $y ə$ ', e.g. ' non- $y / y ə$ ' :--

${ }^{1}$ Following the precedent set by Parts I and II only those exponents of terms in prosodic systems which are phonetically different from each other have been stated. Thus where it appears in the text that no exponents may be stated for a particular prosodic term in a particular prosodic context, it must be understood that the exponents of the prosodic term are in this context phonetically identical with those of the other term(s) in the system. 
A. non-y : (C-) dental nasality, e.g. :-

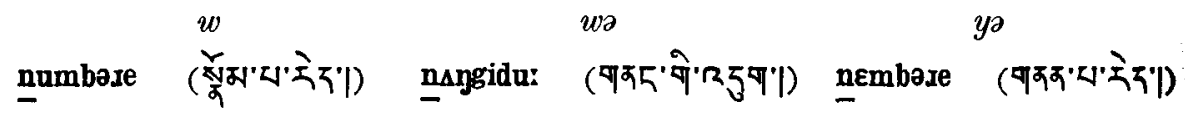

B. non-w : $\left(\mathrm{C}_{2}{ }^{-}\right)$palatal semi vowel, absence of rounding,

$\left(-V_{1}\right)$ absence of rounding, e.g. :-

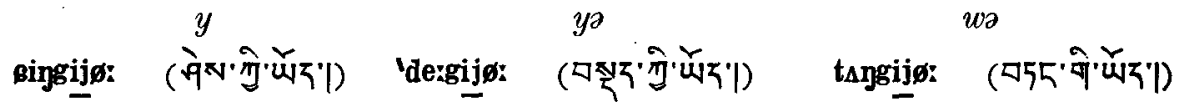

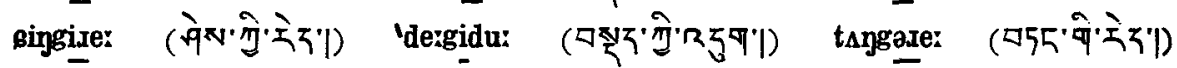

C. non-y/yə : (C-) velarity

$w$

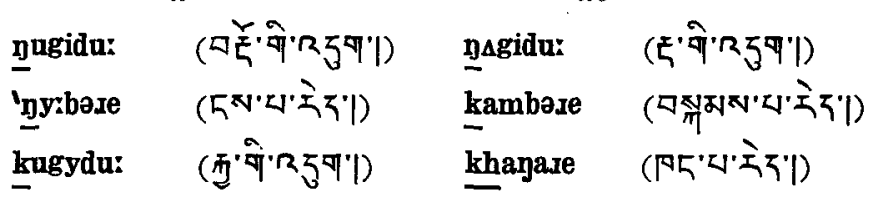

\section{Prosodic and Phonematic Systems}

For $ə \mathrm{~W}$ prosodic and phonematic systems are stated as follows :-

A. 'Prosodic Systems :-

(1) Word : c/o

(2) Syllable Initial (I) : (a) $\mathrm{r} /{ }^{\mathrm{n}} \mathrm{r} ;(b) \mathrm{y} / \mathrm{w}$ (Initial Syllable)

B. Phonematic Terms and Systems :-
(1) $-\mathrm{V}$ -
(2) C- (a) уә: Сa-/C $\beta-/ \mathrm{C} \gamma-$
(b) พә : $\mathrm{C} a-/ \mathrm{C} \beta-/ \mathrm{C} \gamma-$
(Initial Syllable)
$\left(\begin{array}{lll}(, \quad, \quad) \\ (,, \quad, \quad)\end{array}\right.$

(3) $-\mathrm{C}_{\mathrm{a}} /-\mathrm{C}_{\mathrm{b}}$

\section{A. Prosodic Systems}

(1) WORD : c/o

Exponents of c/o may be stated for the initial and medial syllables (CVC, $\mathrm{C}_{1} \mathrm{~V}_{1}$ ). They comprise :-

(a) Medial Syllable

(i) $-V_{1}$ :

$$
\begin{aligned}
& \text { c: a close degree of aperture } \\
& 0: \text { an open , , , }, " \text {, :- }
\end{aligned}
$$

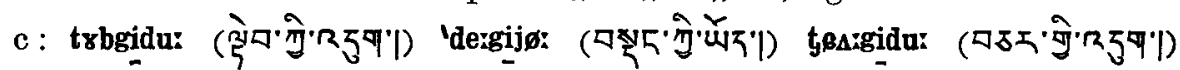

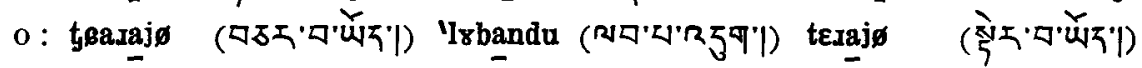

The examples given for ' $\mathrm{o}$ ' above are of ${ }^{n} 1 \mathrm{C}_{1} \mathrm{~V}_{1}$ (see below, Medial and Final Syllables ; Prosodic System of Medial Syllable, $\left.1 /{ }^{n} 1\right)$; none may be given for $\mathbf{1 C}_{1} \mathrm{~V}_{1}$. 
(ii) $\mathrm{C}_{\mathbf{1}^{-}}$:

$$
\text { c: velarity; } \quad 0 \text { : labiality, e.g. :- }
$$

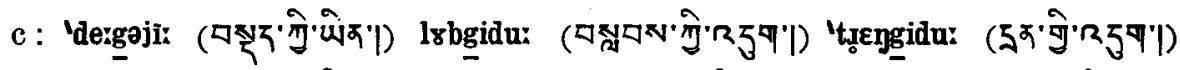

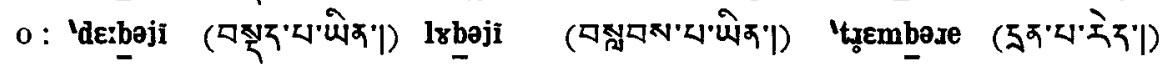

(b) Initial Syllable $(\mathrm{CVC})$

(i) -V-:

The exponents of $c / o$ are stated here in relation to the prosodic system $\mathrm{f} / \mathrm{s}$ (fS/sS, Part I) and to the $-\mathrm{C}$ systems ;

(a) yəW:

$-\mathrm{T}$ :

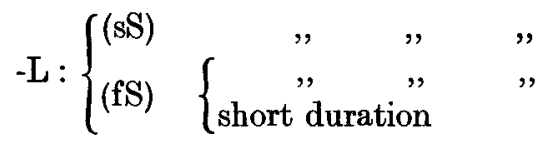

(B) $w a W:$

$-\mathrm{T}:\{$

open degree of aperture

frontness

$-\mathrm{L}:\left\{\begin{array}{l}\text { (sS) open degree of aperture } \\ \text { (fS) }\left\{\begin{array}{l}", " \\ \text { frontness } \\ \text { short duration }\end{array} "\right.\end{array}\right.$

$-K: \begin{cases}\text { (sS) } & \begin{array}{l}\text { open degree of aperture } \\ \text { frontness } \\ \text { short duration }\end{array} \\ \text { (fS) } & \text { open degree of aperture }\end{cases}$

$\left.\begin{array}{l}-\mathrm{M}: \\ -\mathrm{D}:\end{array}\right\}$

(a) уә :

$-\mathrm{T}\left\{\begin{array}{l}\text { thębəje } \\ \text { geibəji } \\ \text { tue:bəji }\end{array}\right.$

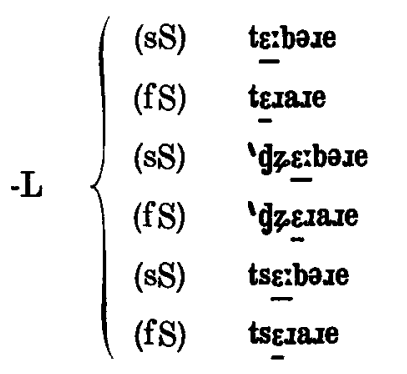

o

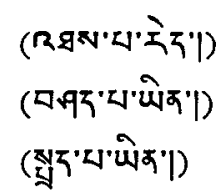

\} (췯ㅁㅁำ

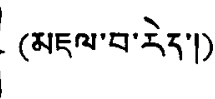

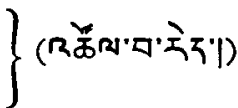

half-open degree of aperture backness

half-open degree of aperture

backness

long duration

half-open degree of aperture

backness

long duration

half-open degree of aperture

backness, e.g.

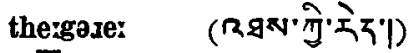

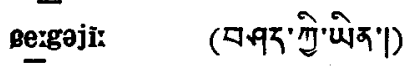

tJęgojĩ

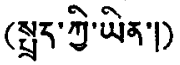

te:geji

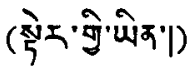

'gzexgəлe:

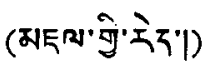

tse:gidu:

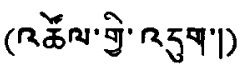




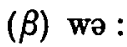

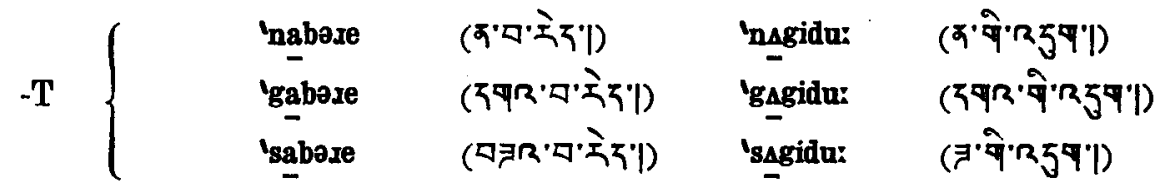

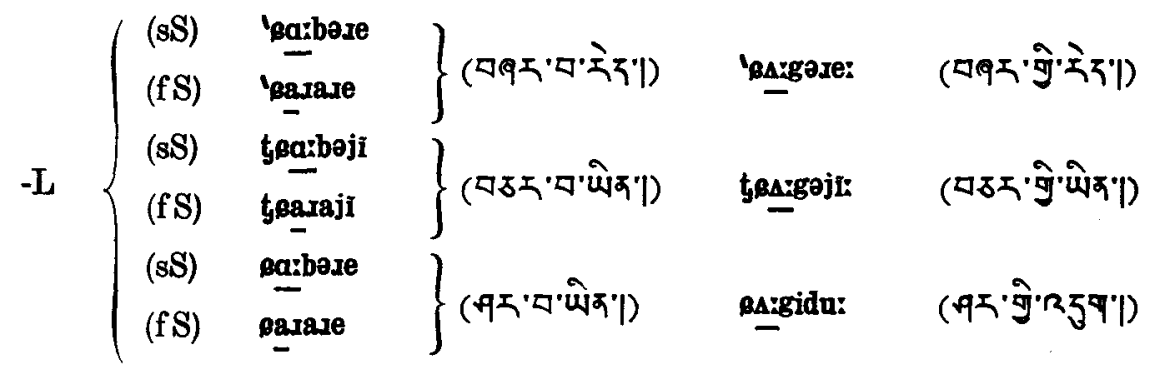

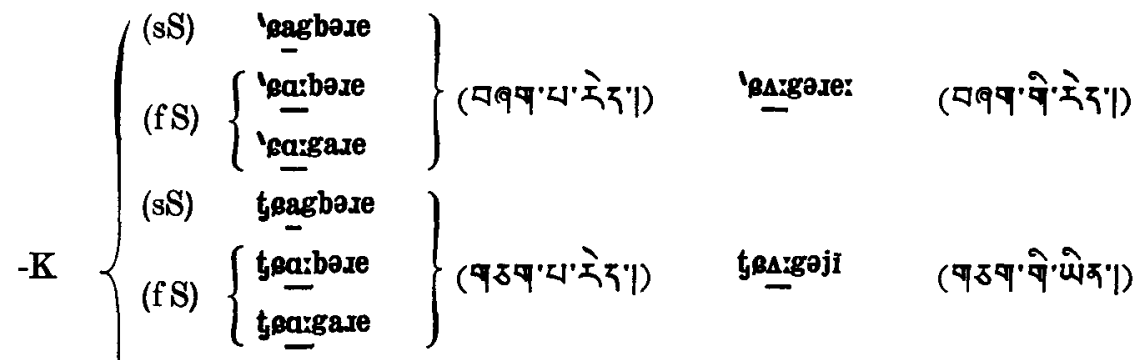

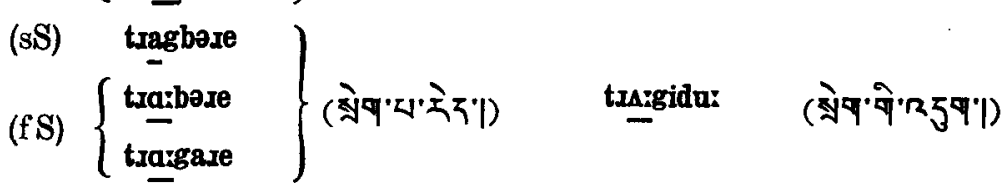

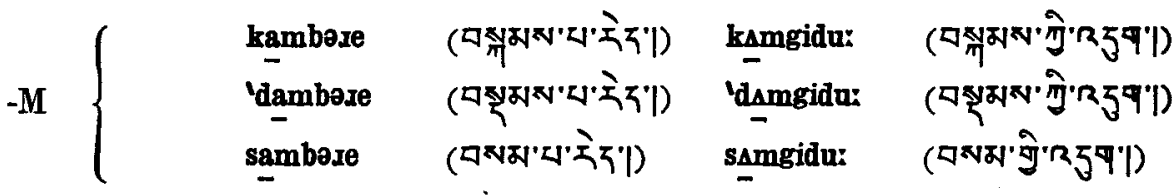

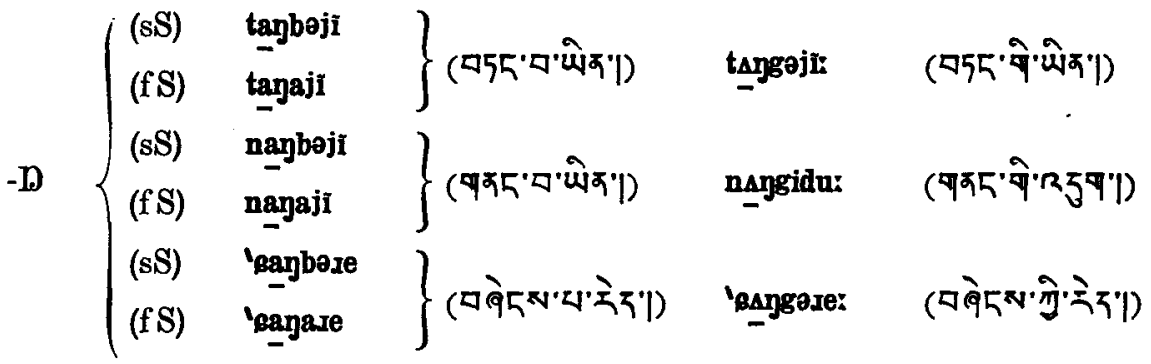

In $\mathrm{sS}$ examples were recorded in which yəW with $-\mathrm{L}$ as final consonant were distinguished phonetically in accordance with an orthographic distinction, i.e. whether the vowel symbol was Rो్ㄱ' 


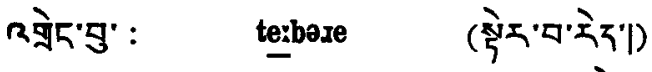

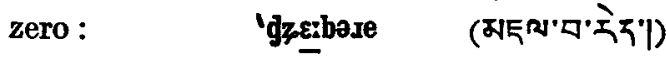

This distinction is thought to be due to the influence of the spelling; there was no phonetic distinction in $\mathbf{f S}$.

(ii) $-\mathrm{C}$ :

For $-\mathrm{C}$ the following exponents of $\mathrm{c} / \mathrm{o}$ may be stated :-

$$
0
$$

$c$

$$
\begin{array}{ll}
-\mathrm{L}(\mathrm{fS}): & \text { friction }(x) \\
-\mathrm{K}: & \text { velarity }
\end{array}
$$$$
-\mathrm{P}:
$$

labiality

$-\mathrm{N}: \quad$ labiality

$-\mathrm{L}(\mathrm{fS})$

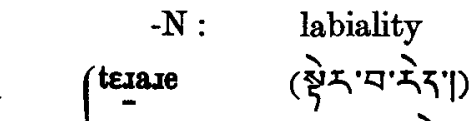

velarity, e.g. :-

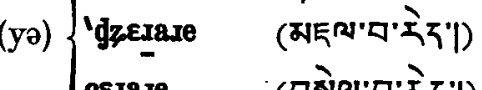

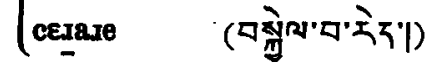

tergaji:

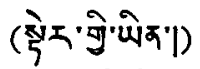

'gzergaje:

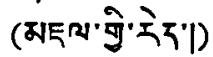

ce:gida:

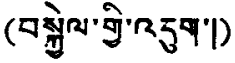

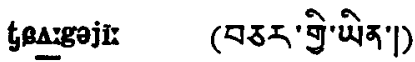

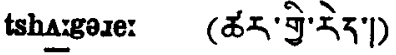

ja:gidu:

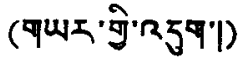

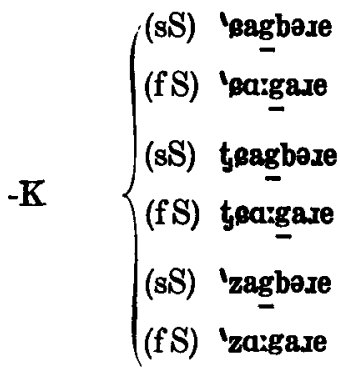

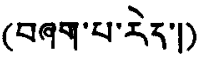

'Baygaje:

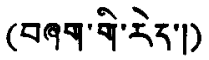

teargidu:

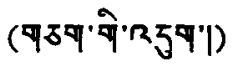

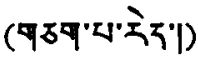

'za:gida:

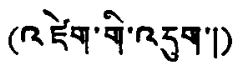

$-P \quad\left\{\begin{array}{l}\text { lrboji } \\ \text { trboxe } \\ \text { 'phrboxe }\end{array}\right.$

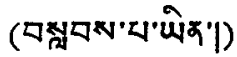

lrbgeji

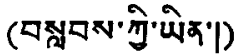

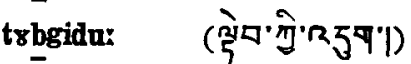

'phrbgidu:

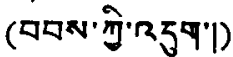

(ロロ・ำ

tengidu:

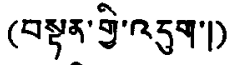

$-\mathrm{N}$

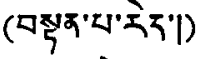

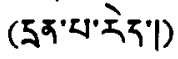

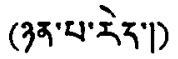

'tūEgidu:

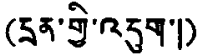

'nengidu:

(अवं छें

(c) Final Syllable $\left(C_{2} \nabla_{2}\right)$

In $\mathrm{fS}_{2}$ - is characterized by the following exponents of $\mathrm{c} / \mathrm{o}$ :-

c: plosion, e.g.

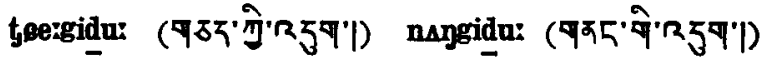

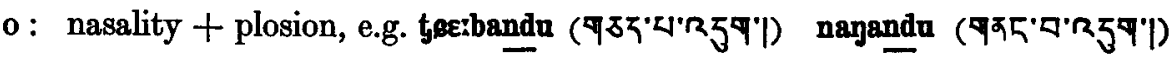


It is of interest that the vowel of yəW in a 'close' prosodic context (cW) may be identical phonetically with the vowel -E- of a $\mathrm{yW}$ in an open prosodic context, (oW) e.g. :-
(a) $-\mathrm{E}-\quad(\mathrm{oW}):$ phe:bəse

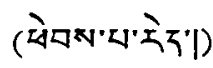

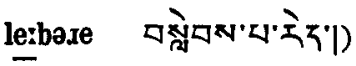
(b) $-\mathrm{y} ə \mathrm{~V}-(\mathrm{cW})$ : thezgexe:

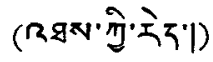

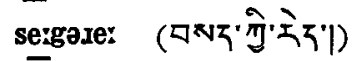

but in comparable prosodic contexts there is no such phonetic identity, e.g. :-
(a) $-\mathrm{E}-\quad(\underline{\mathrm{WW}})$ : phexbəre

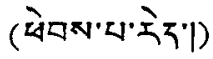

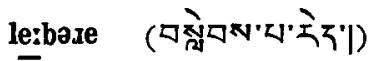
(b) $-\mathrm{y} ə \mathrm{~V}-(\mathrm{oW})$ the:bəre

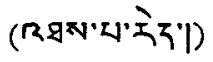

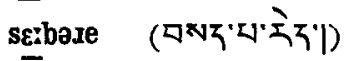

where the $-\mathrm{C}$ term is $-\mathrm{N}$ there is, however, a phonetic identity of vowel-quality as between the vowel of $y \mathrm{~W}$ and $\mathrm{y} \partial \mathrm{W}$ in the 'open' prosodic context, e.g. :-
(a) $\mathrm{yW}$ вembere
(मेक्येंरों)
thembere

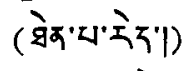

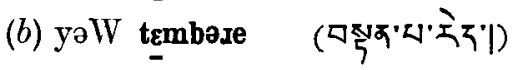
sqmbəre

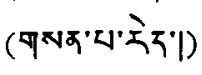

but not in the 'close', e.g. :-

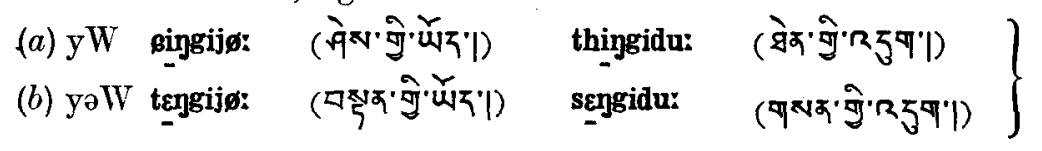

(2) Prosodic Systems of Syllable Initial (I)

Two prosodic systems are recognized for $\mathrm{I}$ in $ə \mathrm{~W}$ just as in $\mathrm{wW}$ and $\mathrm{yW}$ :-

(a) $\mathrm{r} /$ non-r $\left(\mathbf{r} / \mathbf{n}^{\mathrm{r}}\right)$;

(b) $\mathrm{y} / \mathrm{w}$

The exponents of these two systems may characterize both $\mathrm{C}-$ and $-\mathrm{V}-$, i.e. $\mathrm{CV}(\mathrm{C})$.

(a) $\mathrm{r} / \mathrm{n}_{\mathrm{r}}$

The exponents of $\mathrm{r}$ comprise :-

C- : fricative release $(\mathrm{I})$;

the exponents of $\mathrm{n}_{\mathbf{r}}$ comprise :-

(i) C- : A release other than alveolar fricative (x), e.g. alveolar fricative (s), alveolar lateral, plosive, etc.

In yI syllables, lip-spreading.

(ii) -V - : In yI syllables, a greater degree of frontness in wəW, and of closeness in yəW, than is appropriate to ' $r$ ', e.g. :-

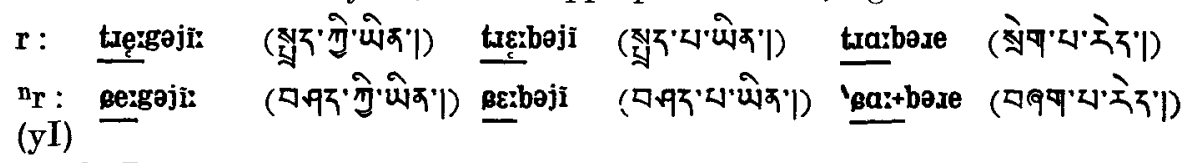

(b) For the stating of ${ }^{n_{r} I}$ a two-term system $(y / w)$ is also required for $ə \mathrm{~W}$. The phonetic exponents of $y / w$ comprise :-

(i) $-\mathrm{V}-$ :

$y$ : a greater degree of closeness ( $y \partial W$ ) and frontness (wəW) than is appropriate to ' $\mathrm{w}$ ' in otherwise the same prosodic context, e.g. :- 


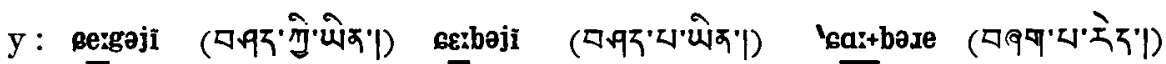

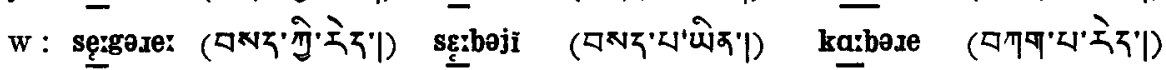

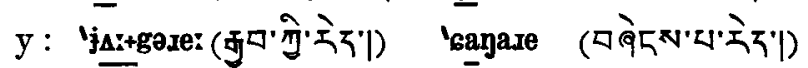

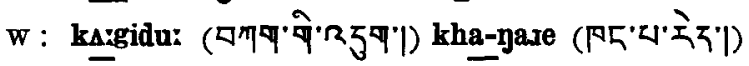

(ii) $\mathrm{C}-$ :

$y:$ lip-spreading, articulations as below, in relation to the $\mathrm{C}$ - systems (§ B 2a)

w : articulations as below, in relation to the C- systems (§ B 2a) :-

$y$

P- : Alveolar (occlusion) + fricative release

$\mathrm{T}-:\left\{\begin{array}{c}(1 \mathrm{~W}) \text { Alveolar (occlusion) }+ \text { frica- } \\ \text { tive release } \\ (2 \mathrm{~W}) \text { Alveolar friction }(\mathrm{z})\end{array}\right.$

K- : Palatal (plosion)

Q- : Palatal semi-vowel

L- : (Alveolar) lateral

S- : Alveolo-palatal (friction)

N-: Palatal (nasality)

Labial (occlusion) + plosive release

Dental (occlusion) + plosive release

Velar (plosion)

Absence of consonant articulation

(Alveolar) friction (I)

Alveolar (friction) (s)

Dental

Labial (nasality), e.g.

Velar

$y$

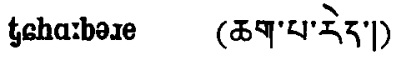

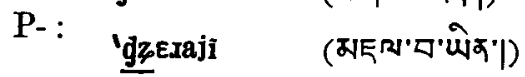

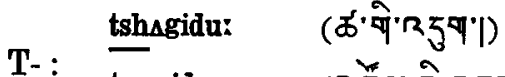

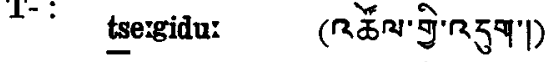

K- :

'jrbese

cesase

jembəxe

Q- : 'je:gidu:

Isbgijø:

L- :

'sayase

S- : cergidu:

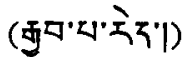

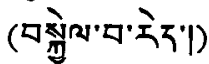

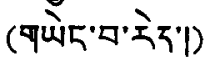

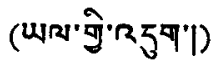

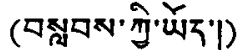

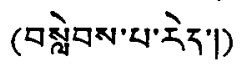

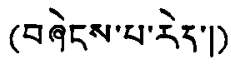

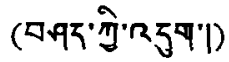

$w$

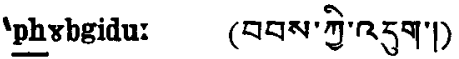

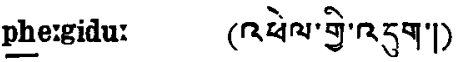

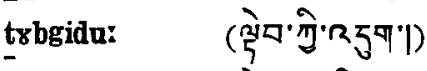

tesajĩ

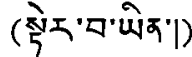

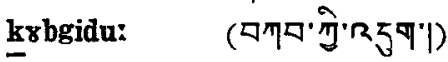

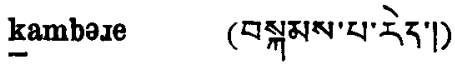

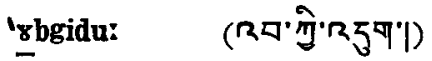

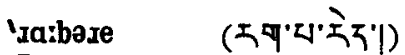

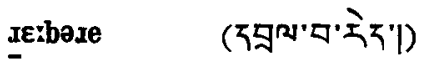

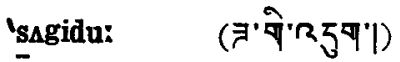

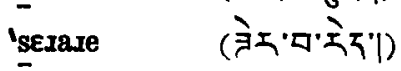

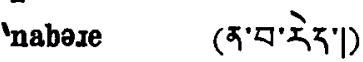




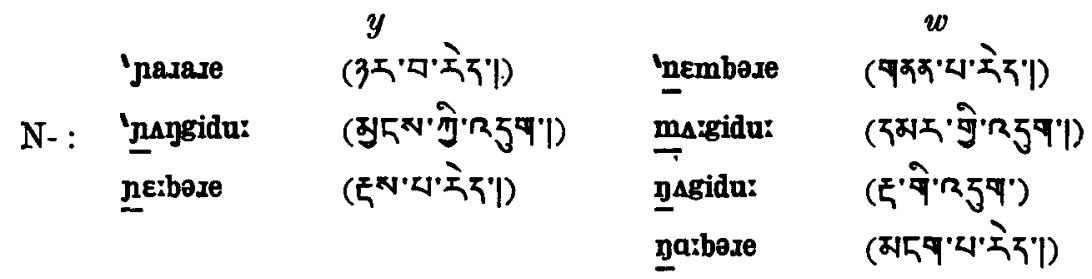

B. Phonematic Systems (Initial Syllable - CVC)

(1) $-\mathrm{V}-$ :

For the exponents of $-\mathrm{V}$ - in $ə \mathrm{~W}$ one must take into account the following prosodic systems :-
(a) $\mathrm{f} / \mathrm{s}$ (Sentence- $\mathrm{S}$ )
Part I
(b) $\mathrm{c} / \mathrm{o}$ (Word-W)
$\S$ II A 1
(c) $\mathrm{y} / \mathrm{w}$ (Syllable-S)
$\S \mathrm{I}$
(d) $\mathbf{r} / \mathbf{n}_{\mathbf{r}}$
(e) $\mathrm{y} / \mathrm{w}\}$ (Syllable Initial-I) \& II A 2,

and the phonematic systems stated below (-C, § B 2b).

$-\mathrm{V}-$ :

$$
\begin{aligned}
& \text { yS/cW/rI/(-T/-L) : ę: tuẹggoji: } \\
& \text {, , , }-\mathrm{N}: \quad \varepsilon \text { 'tJęgida: } \\
& \text { ", }{ }^{n_{r} I / y I /(-T /-L) ~: ~ e: ~ c e r g e j i ~} \\
& \text {, , , , }-\mathrm{N}: \quad \varepsilon \text { 'reggidu: } \\
& \text {, , , wI } /(-\mathrm{T} /-\mathrm{L}): \quad \text { e: tẹggese: } \\
& \text { ", ", , }-\mathrm{N}: \quad \varepsilon \quad \text { tengəji: } \\
& \text {, oW } / \mathrm{rI} /-\mathrm{T} \text { : }
\end{aligned}
$$

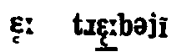

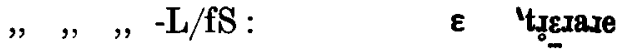

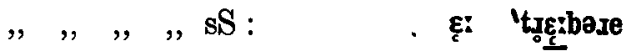

$$
\begin{aligned}
& , \quad, \quad,-\mathrm{N} \text { : } \\
& \varepsilon \text { 'țદmbaje } \\
& \text {, , } \mathrm{n}_{\mathrm{r}} \mathrm{I} / \mathrm{y} \mathrm{I} / \mathrm{-T} \text { : } \\
& \text { E: ce:boji } \\
& , \quad, \quad, \quad, \quad-\mathrm{L} / \mathrm{fS} \text { : } \\
& \varepsilon \text { 'gzeraje } \\
& ,,,,,, \quad \text { sS : } \\
& \text { e: 'dze:bəre } \\
& \text {, , , , },-\mathrm{N} \text { : } \\
& \text {, , , } \quad \text { wI/-T : } \\
& \varepsilon \text { 'лعmbase } \\
& \text { ๕̨: sę:bəre }
\end{aligned}
$$

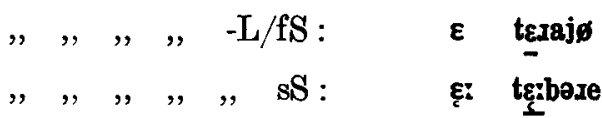

$$
\begin{aligned}
& \text { ", , , }, \quad-\mathrm{N}: \quad \varepsilon \quad \text { temboji }
\end{aligned}
$$

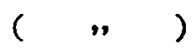

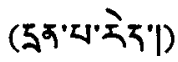

$$
\begin{aligned}
& \text { (घभर'चयेव') }
\end{aligned}
$$

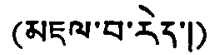

$$
\begin{aligned}
& \text { ( } ")
\end{aligned}
$$

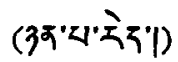

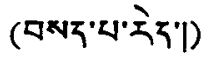

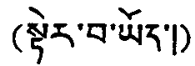

$$
\begin{aligned}
& \text { ( } ") \\
& \text { (एश्रू'पयेका) }
\end{aligned}
$$




\begin{tabular}{|c|c|c|c|c|c|c|}
\hline \multicolumn{4}{|c|}{$\mathrm{wS} / \mathrm{cW} / \mathbf{r I} /(-\mathrm{T} /-\mathrm{M} /-\mathrm{D}):$} & $\Delta$ & trungida: & (श्रॉर्वे \\
\hline " & , & , & $(-\mathrm{L} /-\mathrm{K}):$ & $\Delta:$ & trusgidu: & (खे \\
\hline " & ", & " & $-\mathbf{P}:$ & 8 & trrybgidu: & (R⿴囗十 \\
\hline " & $"$ & & $\mathrm{I} / \mathrm{y} \mathrm{I} /(-\mathrm{T} /-\mathrm{M} /-\mathrm{D}):$ & $\Delta$ & tshugidu: & \\
\hline$"$ & " & $"$ & $, \quad(-\mathbf{L} /-\mathbf{K}):$ & $\Delta i+$ & tosi+gajĩ: & \\
\hline$"$ & " & $"$ & $" \quad-\mathbf{P}:$ & 8 & l8bgəji: & $(\square \times)$ \\
\hline ", & ", & ", & $\mathrm{wI} /(-\mathrm{T} /-\mathrm{M} /-\mathrm{D})$ : & $\boldsymbol{\Delta}$ & tajgidu: & $\Psi^{\prime} \mid$ \\
\hline$"$ & " & $"$ & $, \quad(-\mathrm{L} /-\mathrm{K}):$ & $\Delta:$ & mägidu: & $41)$ \\
\hline$"$ & $"$ & $"$ & $" \quad-\mathbf{P}:$ & 8 & 'phzbgidu: & (ロロ \\
\hline$"$ & $\mathrm{oW}$ & $/ \mathrm{rI}$ & $/(-\mathrm{T} /-\mathrm{M} /-\mathrm{D}))$ & a- & tra-yare & \\
\hline " & , & , & $\left.\begin{array}{r}-\mathrm{L} / \mathrm{fS}: \\
\mathrm{SS}:\end{array}\right\}$ & nol & ne recorded & \\
\hline " & , & $"$ & $-\mathbf{K} / \mathbf{f S}:$ & $\mathbf{a x}^{\mathbf{x}}$ & trazbare & (ख्रेष'ए' \\
\hline ", & $"$ & $"$ & " $\quad \mathrm{sS}:$ & $\mathbf{a}$ & tragbaxe & ( $\quad$ " \\
\hline " & $"$ & , & $-\mathrm{P}:$ & 8 & trg8bəxe & (R⿴囗十 \\
\hline "' & " & & $\mathrm{I} / \mathrm{y} \mathrm{I} /(-\mathrm{T} /-\mathrm{M} /-\mathrm{D}):$ & $\mathbf{a}$ & 'sayare & खवे \\
\hline$"$ & $"$ & ", & $, \quad-\mathrm{L} / \mathrm{fS}:$ & $\mathbf{a}$ & tgeraji & (ロঠエ'5 \\
\hline$"$ & $"$ & $"$ & $, \quad, \quad$ sS : & a:t & tgca:+bojĩ & ( \\
\hline "’ & $"$ & $"$ & $, \quad-\mathbf{K} / \mathbf{f S}:$ & $a_{*}+$ & tocax+bəji & 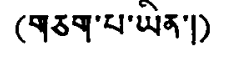 \\
\hline$"$ & $"$ & " & $, \quad, \quad$ sS : & $\mathbf{a}$ & toagbaji & ( \\
\hline$"$ & ", & ", & $, \quad-\mathbf{P}:$ & 8 & lzbəji & (잼ㅁ \\
\hline$"$ & ", & $"$ & 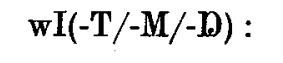 & a- & 'ga-bəدe & $(59 \pi$ \\
\hline " & " & $"$ & $, \quad-\mathbf{L} / \mathrm{fS}:$ & a- & pa-race & ( 此原' \\
\hline$"$ & $"$ & $"$ & ",$\quad$ SS : & a: & paxbose & ( \\
\hline$"$ & $"$ & $"$ & $, \quad-\mathbf{K} / \mathbf{f S}:$ & $\mathbf{a}:$ & kasboxe & $(\square \pi \mathbf{4})$ \\
\hline$"$ & $"$ & $"$ & $, \quad, \quad$ sS : & a- & ka-gbaje & ( \\
\hline "' & $"$ & $"$ & , $\quad-\mathbf{P}:$ & $\mathbf{8}$ & k8baxe & (ロクロ \\
\hline
\end{tabular}

(2) C Systems

(a) C-Systems

Separate C- systems need to be stated for yəW and wəW.

(i) $\mathrm{waW}$

(a) $\mathrm{C}_{a^{-}}$: 
The $\mathrm{C}_{a^{-}}$-system is that which is appropriate to words for which the following prosodic systems have to be taken into account :-

$\mathrm{rC}_{a^{-}}$:

$$
\left.\begin{array}{l}
\mathrm{h} / \mathrm{nh}_{\mathrm{h}} \\
\mathrm{v} / \mathrm{n}_{\mathrm{v}}
\end{array}\right\} \mathrm{r} / \mathrm{n}_{\mathrm{rC}} \mathrm{C}
$$

In examples in rI ( $\$$ A 2a) no system may be stated for $\mathrm{C}_{a^{-}}$. $\mathrm{C}_{a^{-}}$is characterized by alveolar-affricate (tI) articulation :-

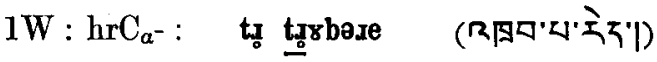

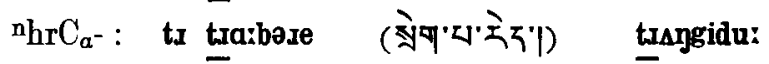

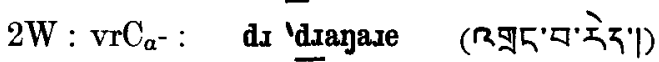

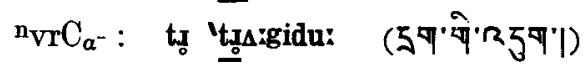

Where, however, the syllable-initial prosody is ${ }^{{ }^{n}} r$, and the $y / w$ prosodic system may be set up for the syllable initial, i.e.

$$
\mathrm{n}_{\mathrm{r}}\left\{\begin{array}{l}
\mathrm{h} / \mathrm{n}_{\mathrm{h}} \\
\mathrm{v} / \mathrm{n}_{\mathrm{v}}
\end{array}\right\} \mathrm{y} / \mathrm{wC}-
$$

a three-term system $\mathrm{P}-/ \mathrm{T}-/ \mathrm{K}$ - is recognized :-

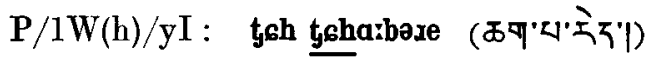

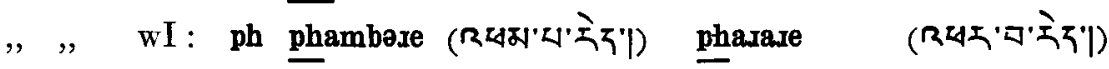

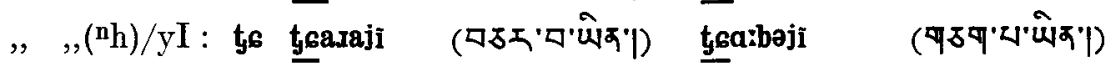

$$
\begin{aligned}
& , \quad, \quad \text { wI : p pargidu: ( }
\end{aligned}
$$

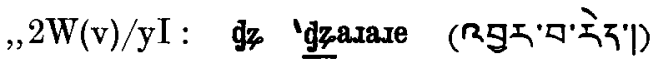

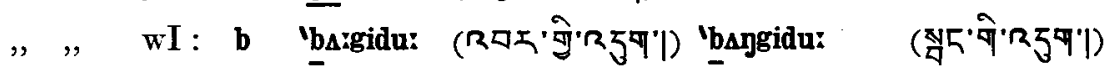

$$
\begin{aligned}
& \text {, ,( }\left(\mathrm{n}_{\mathrm{v}}\right) / \mathrm{yI}: \text { none recorded }
\end{aligned}
$$

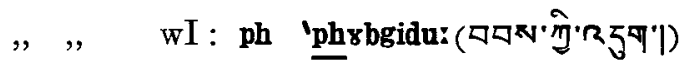

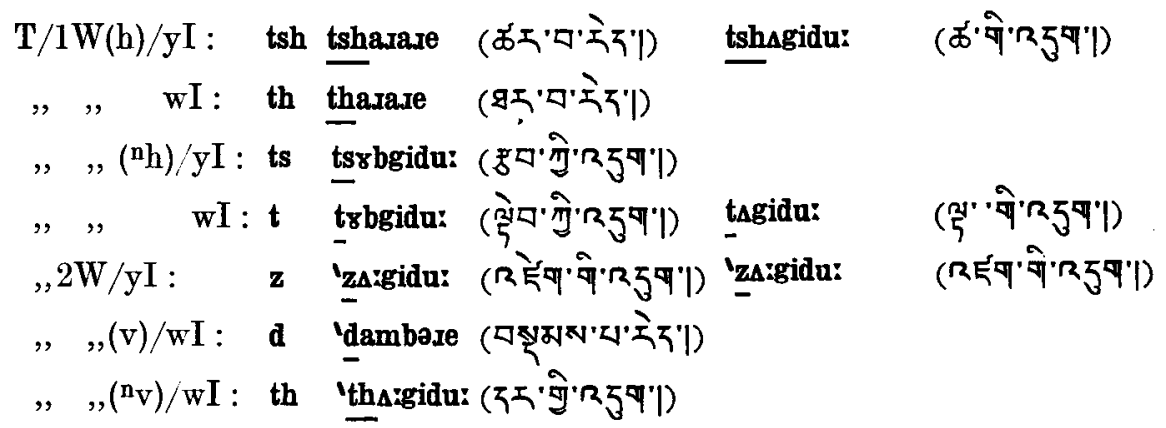

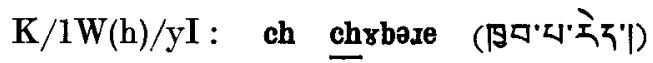

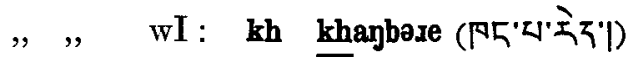

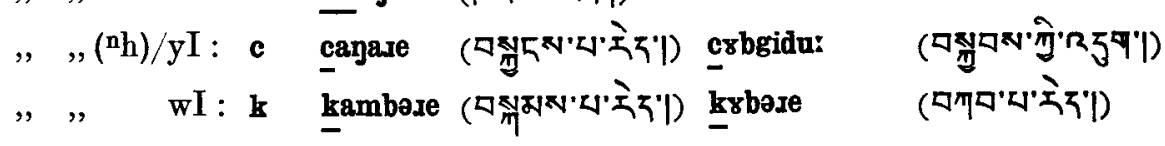




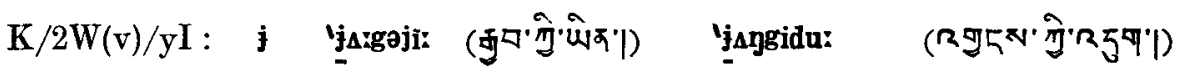

, , wI: $\quad \mathrm{g} \quad$ 'g

$, \quad,\left(\mathrm{n}_{\mathrm{v}}\right) / \mathrm{yI}: \quad$ none recorded

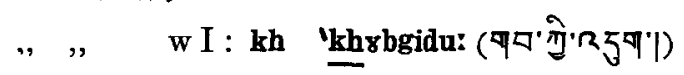

(ק) $\mathrm{C}_{\beta-}$ :

In syllables where the $y / w$ prosodic system $(\$ A 2 a)$ may be stated, i.e. $\mathrm{y} / \mathrm{wC}-$, a four-term system is recognized :-

$$
\text { Q-/L-/S-/D-, e.g. :- }
$$

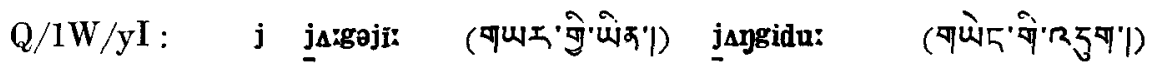

, , wI : none recorded

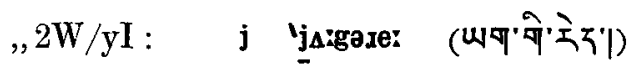

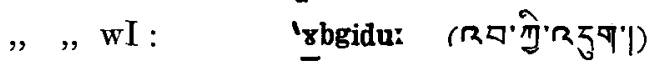

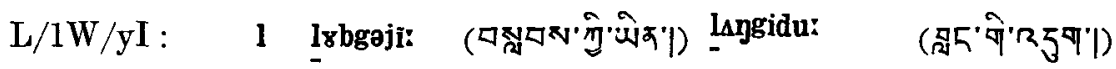

, , wI : none recorded

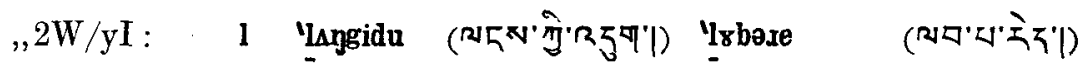

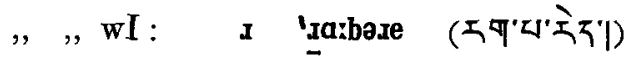

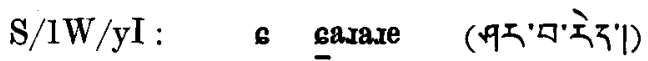

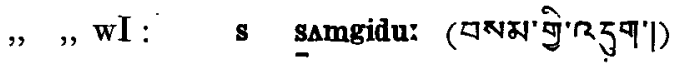

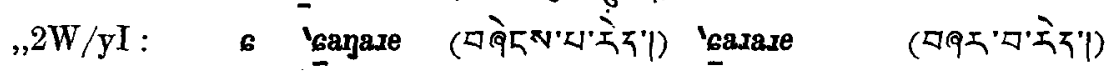

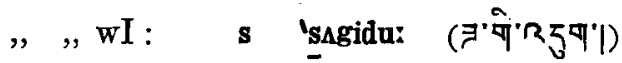

$\mathrm{D} / \mathrm{IW} / \mathrm{yW}$ : none recorded

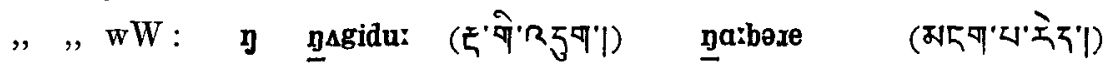

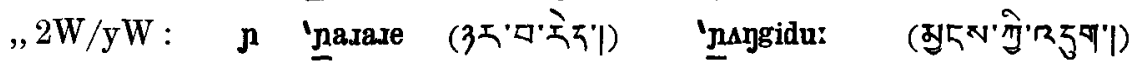

, , wW : none recorded

In addition to the two examples given for $\mathrm{L} / 1 \mathrm{~W} / \mathrm{yI}$ there were two recorded

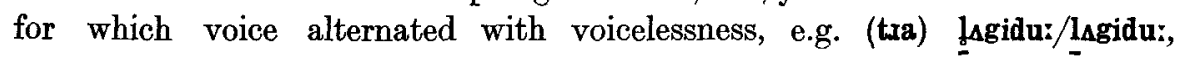

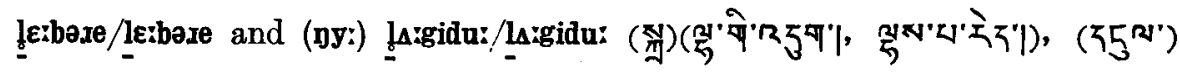

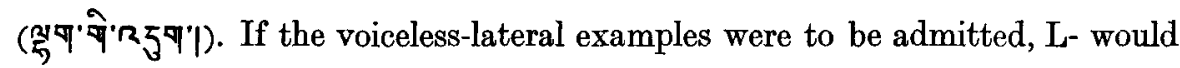
have to be removed from the $\mathrm{C}_{\beta}$ - system ; they have here been disregarded in favour of the more often recorded voiced-lateral examples.

$(\gamma) \mathrm{C}_{\gamma^{-}}$:

The nasal-initial system comprises the two terms $\mathrm{M}-\mathrm{N}$-, for wI syllables, e.g. :- 


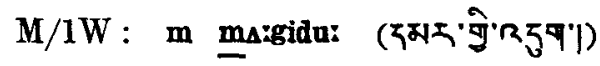

„2W : none recorded

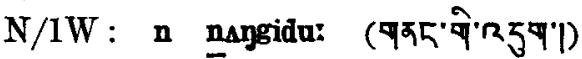

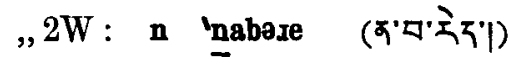

(ii) $\mathrm{y}_{\partial \mathrm{W}}$

For yəW the C- systems stated for woW are not valid. The C- systems in yoW are not identical with those of $y W$, but resemble them. They comprise :-

(a) $\mathrm{C}_{a^{-}}$:

The C-system is that which is appropriate to words for which the following prosodic systems have to be taken into account :-

$\mathrm{rC}_{a^{-}}$

$$
\left.\begin{array}{l}
\mathrm{h} /{ }^{n} \mathrm{~h} \\
\mathrm{v} / \mathrm{n}_{\mathrm{v}}
\end{array}\right\} \mathrm{r} / \mathrm{n}_{\mathrm{rC}} \mathrm{C}
$$

In examples in $\mathrm{rI}$ ( $\$ \mathrm{~A} 2 \mathrm{a}$ ) $\mathrm{C}_{\boldsymbol{a}^{-}}$is characterized by alveolarity with fricative (x) release, e.g. :-

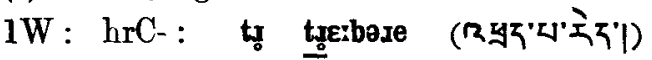

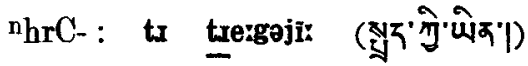

$2 \mathrm{~W}:$ vrC- : none recorded

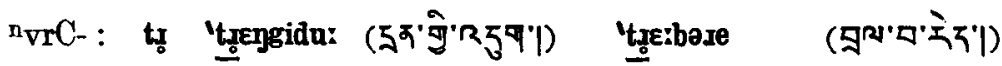

In syllables in ${ }^{n_{r} I}$, where the $y / w$ prosodic system may be stated for the syllable initial, i.e. :-

$$
\mathrm{n}_{\mathrm{r}}\left\{\begin{array}{l}
\mathrm{h} / \mathrm{n}_{\mathrm{h}} \\
\mathrm{v} / \mathrm{n}_{\mathrm{v}}
\end{array}\right\} \mathrm{y} / \mathrm{wC}-
$$

A two-term system, $\mathrm{P} / \mathrm{T}$, may be stated, as opposed to the three-term system $(\mathrm{P} / \mathrm{T} / \mathrm{K})$ of wəW.

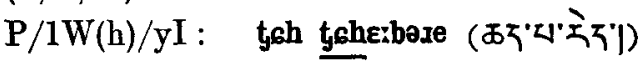

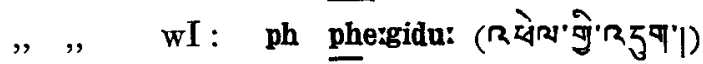

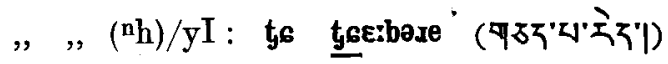

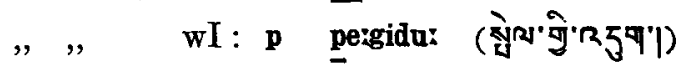

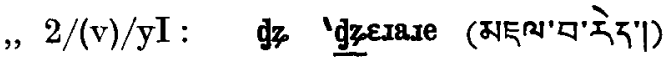

wI : none recorded

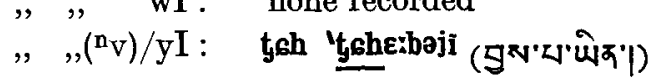

", wI : none recorded

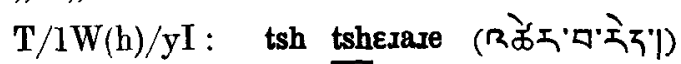

wI: th the:base (Rgavు'主们)

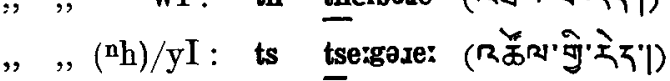

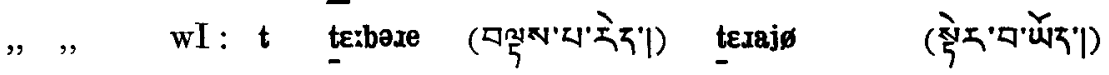


$\mathrm{T} / 2 \mathrm{~W} / \mathrm{yI}: \quad$ none recorded

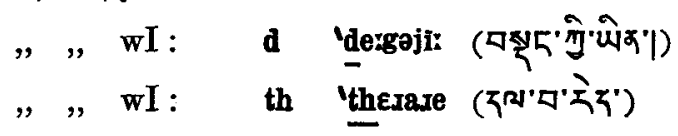

Where the $\mathrm{C}$ - term is $\mathrm{K}$-, i.e. :-

$$
\mathbf{n}_{\mathbf{r}}\left\{\begin{array}{l}
\mathrm{h} / \mathbf{n}_{\mathrm{h}} \\
\mathbf{v} / \mathbf{n}_{\mathrm{v}}
\end{array}\right\} \mathrm{yC}_{\boldsymbol{\alpha}^{-}}
$$

the following are the exponents of $\mathrm{K}-$ :-

\begin{tabular}{|c|c|c|c|c|c|}
\hline $\mathrm{K} / \mathrm{1W}(\mathrm{h}):$ & ch & chejare & 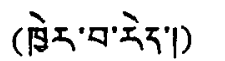 & chejaje & 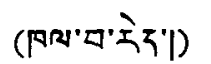 \\
\hline$,, \quad,\left({ }^{n} h\right):$ & c & sase & (亡ेz'1) & cejare & 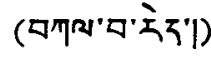 \\
\hline ,2W(v): & $\dot{j}$ & & 文保1 & 'jeraje & Fil \\
\hline$", \quad\left({ }^{n} v\right)$ & ch & & Rรुप'|) & & \\
\hline
\end{tabular}

( $\beta$ ) $\mathrm{C}_{\beta^{-}}$:

In words for which the prosodic systems $\mathrm{h} /{ }^{\mathrm{h}} \mathrm{h}$ and $\mathrm{v} /{ }^{\mathrm{n}} \mathrm{v}$ are inapplicable, but for which $y / w$ may be stated for the syllable initial, i.e. $y / w C-$, a two-term system, L-/S-, may be stated. The exponents of $\mathrm{L}_{-}$and $\mathrm{S}$ - are :-

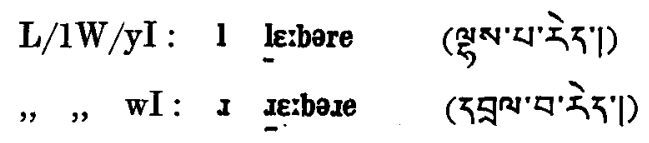

,2W/yI : none recorded

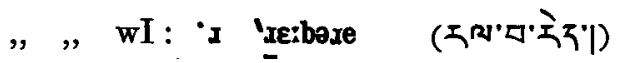

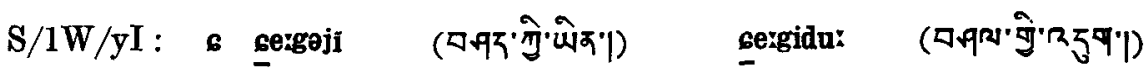

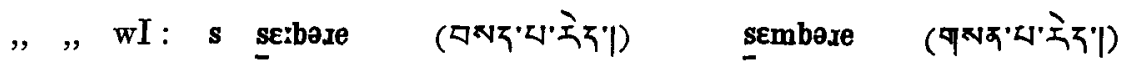

„, $\partial \mathrm{W} / \mathrm{yI}: \quad$ none recorded

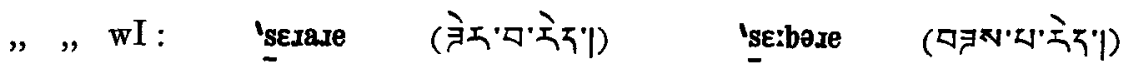

The example of $\mathrm{L} / 1 \mathrm{~W} / \mathrm{yI}$, leibere, was also recorded as levbere, the former being the more frequently recorded of the two. The form with the voiceless-lateral initial has been excluded from the present study. It could not be stated in the same terms as the form with voiced lateral initial.

The $\mathrm{Q}$ - term is not, as in woW, included in the same system as $\mathrm{L}$-/S-, but in a system for which the $\mathrm{y} / \mathrm{wI}$ system is not appropriate, i.e. $\mathrm{C}_{\gamma^{-}}$, together with $\mathrm{D}$-. The exponents of $\mathrm{Q}$ - are :-
$\mathrm{Q} / \mathrm{1W}$ :
j jembəre

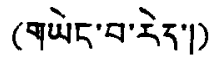
, $2 \mathrm{~W}$ :
j jexase

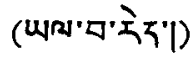


$\mathrm{D}$ - in this prosodic context resembles $\mathrm{K}$-; together with Q- it is confined to yI syllables, e.g. :-
D) $1 \mathrm{~W}$ :
n $\underline{\text { nesaje }}$

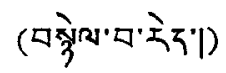
ñ $\varepsilon$ bəre

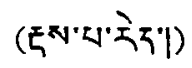
, $2 \mathrm{~W}$ :
s 'ñemboxe

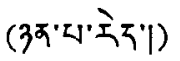
'nergede:

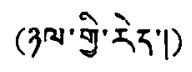

$\mathrm{N}$ - is confined to wI syllables :-
$\mathrm{N} / 1 \mathrm{~W}$ :
n nembəje

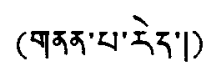
„2W : none recorded

It is surprising that labiality is not a co-articulation of nasality in this prosodic context, for it is in $\mathrm{yW}$, but no examples of initial-labial nasality were recorded. The way in which nasality is stated in oW differs considerably from the statements made for it in $\mathrm{wW}$ and $\mathrm{yW}$. The aW type of statement has been adopted in order to avoid having to treat the alternation of velar with palatal

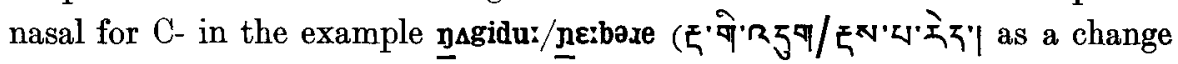
of consonant. In the above statement the $\mathrm{C}$ - term $\mathrm{D}$ - is preserved in both yoW and wəW, but with a change of prosody, from the $\mathrm{y} / \mathrm{wC}$ - system $\left(\mathrm{C}_{\beta}-\right)$ to a system for which $\mathrm{y} / \mathrm{wI}$ are not statable $\left(\mathrm{C}_{\gamma^{-}}\right)$. In $\mathrm{C}_{\gamma^{-}} \mathrm{D}$ - is limited to $\mathrm{yI}$ syllables, its phonetic exponent being therefore palatal nasality. The two forms may be symbolized as follows :-

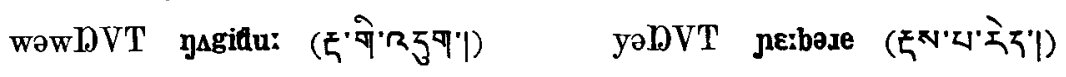

(b) -C Systems

As in the case of the $\mathrm{C}$ - systems the -C systems must be stated with reference to the prosodic system of the initial syllable of $ə W$, i.e. $y / w$, hence wəW/yəW. Certain consonants may be stated for both, while others are restricted to one or the other, e.g. :-
(i) ${ }^{\circ} \mathrm{W} / \mathrm{y} \partial \mathrm{W}: \mathrm{T}, \mathrm{L}$.
(ii) yəW :
N.
(iii) $\mathrm{w}$ W :
$\mathrm{P}, \mathrm{K}, \mathrm{M}, \mathrm{D}$.

The exponents of the -C systems require a statement in terms of $\mathrm{cW}$ and $\mathrm{oW}$. The exponents of the $-\mathrm{C}_{\mathrm{a}}$ system are :-

$c$

$-\mathrm{T}$ : Absence of consonant articulation

$-\mathrm{L}$ :
Absence of consonant articulation

$\left\{\begin{array}{c}\text { Alveolar friction } \\ \end{array}\right.$

1 An interesting contrast with LT was observed for the Tsang dialect of Tibetan. Where in LT alveolar friction ( $I$ ) was the sole recorded exponent of $-L$ in both $y F$ and $w F$ alike, in Tsang Tibetan alveolar friction (x) was recorded for $\mathrm{wF}$ and an alveolar lateral articulation for $\mathrm{yE}$, e.g.
$-\mathrm{L}\left\{\begin{array}{l}\mathrm{wF}: \text { kujaxe } \\ y \mathrm{~F}: \text { kylaji }\end{array}\right.$

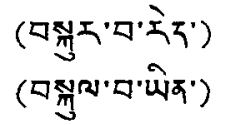
'dzarase

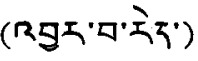

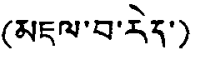


The exponents of the $-C_{b}$ system are :-

-P : Labial occlusion Absence of consonant articulation

-K: Absence of consonant articulation $\left\{\begin{array}{c}\text { Velar plosion } \\ ,, ", ",\end{array}\right\}$

-M : Labial nasality

-D: Velar ,

Labial nasality

Velar "

Where nasality characterizes $-\mathrm{C}$ in a $\mathrm{yF}$ syllable $(-\mathrm{N}$, as opposed to $-\mathrm{M} /-\mathrm{D})$ its exponents are :-
$-\mathrm{N}$ :
velar nasality
labial nasality

In the examples below the phonological structure of the initial syllable, together with the relevant prosodies of the word, is given in the right-hand column.

$$
\text { o }
$$

(i) $-\mathrm{T}$ (พə) :

\begin{tabular}{|c|c|c|c|}
\hline $\begin{array}{l}\text { 'nabəje } \\
\text { 'gabəze }\end{array}$ & 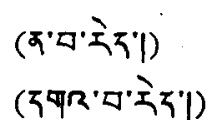 & $\begin{array}{l}\text { 'nagidu: } \\
\text { 'gagidu: }\end{array}$ & 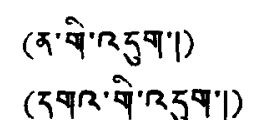 \\
\hline
\end{tabular}

(yə) :

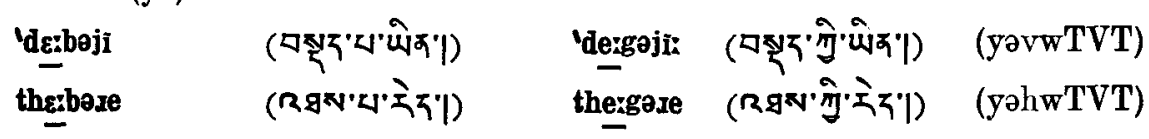

(ii) $-\mathrm{L}(\mathrm{wo})$ :

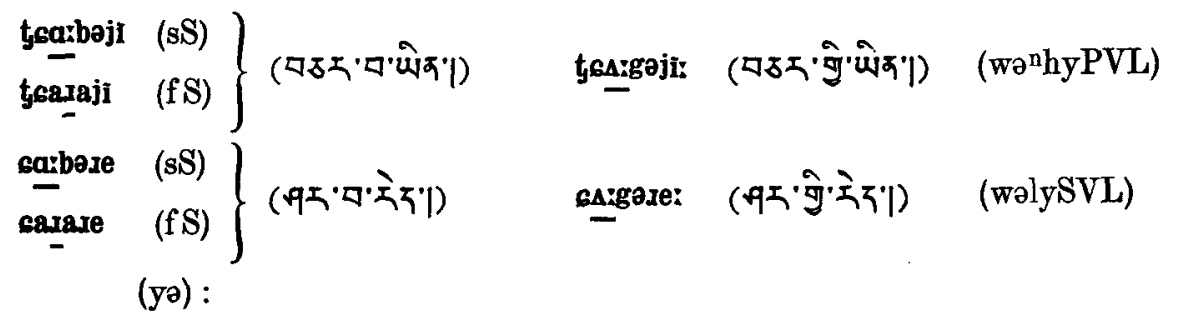

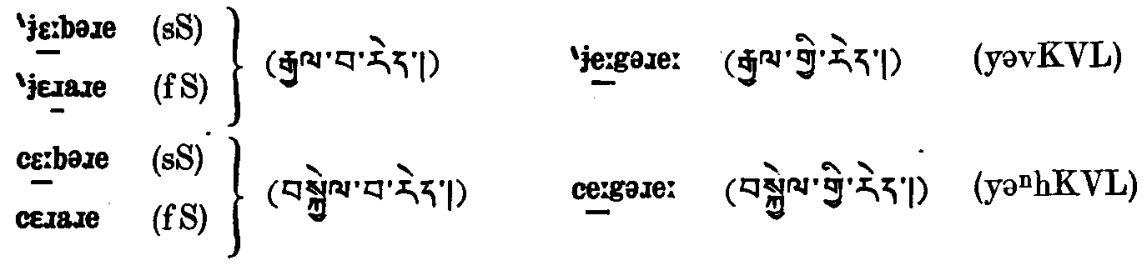

(iii) $-\mathbf{P}$ :

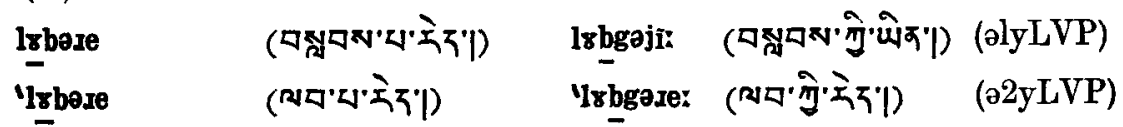


(iv) $-\mathrm{K}$ :
tyagbəدe (SS)
tga:baxe (fS)
(पषणमㄷำ

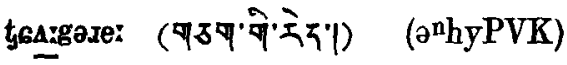
treaigare (fS)
$\left.\begin{array}{ll}\text { tragboxe } & (\mathrm{sS}) \\ \text { traibere } & (\mathrm{fS}) \\ \text { traigare } & (\mathrm{fS})\end{array}\right\}$

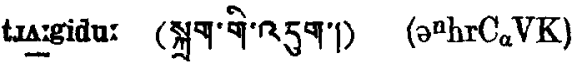
(v) $-\mathrm{M}$ :

\section{sambexe \\ 'dambore}

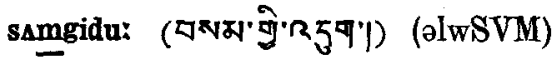

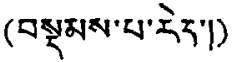

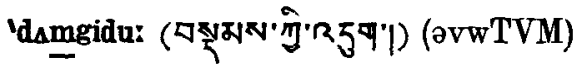
(vi) $-\mathrm{D}$ :

\section{nanbere \\ nanase \\ lanbore \\ 'lanare}

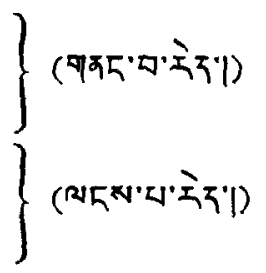

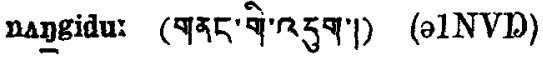

$$
\begin{aligned}
& \text { 'Langidu: ( (25 }
\end{aligned}
$$
(vii) $-\mathrm{N}$ :

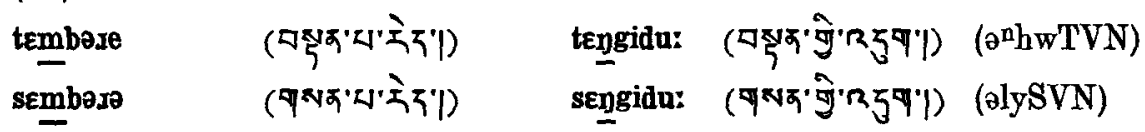

One or two instances have been recorded of a phonetic exponent of $-M$ (in $\mathrm{cW}$ ) other than labial nasality, i.e. velar nasality, e.g. :-

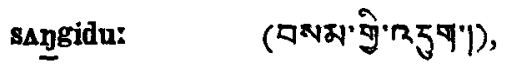

and of - $\mathrm{D}$ (in oW) other than velar nasality, i.e. labial nasality, e.g. :-

tambore

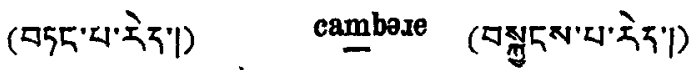

The prosodic and phonematic systems of the medial and final syllables are stated in the following section.

\section{MEDIAL AND FINAL SYLLABLES; PROSODIC AND PHONEMATIC SYSTEMS}

Much of the material now to be stated for the medial $\left(C_{1} V_{1}\right)$ and final $\left(\mathrm{C}_{2} \mathrm{~V}_{2}\right)$ syllables has been stated above under the heading of the prosodic systems of the word $y / w / \theta$ and $c / o$, especially as regards the medial syllable; but the phonematic systems of these two syllables have so far gone unstated, for the reason that they are constant in $\mathrm{yW}, \mathrm{wW},{ }_{\partial \mathrm{W}}$, and in $\mathrm{cW}$ and $\mathrm{oW}$ alike. In the initial syllable (CVC) the systems stated for $\mathrm{C}-,-\mathrm{V}$-, and $-\mathrm{C}$ were related to the prosodic system $\mathrm{y} / \mathrm{w} / \mathrm{\partial}$, and were stated in terms of it. There are in addition to 
the as yet unstated phonematic systems of the medial and final syllables, prosodic systems statable for these two syllables but not for the initial syllable. The form of statement adopted for the medial and final syllables is :-

I. Prosodic Systems

A. Medial and Final syllables $\left(\mathrm{C}_{1} \mathrm{~V}_{1}+\mathrm{C}_{2} \mathrm{~V}_{2}\right)$

B. (1) Medial Syllable

(2) Final Syllable

II. Phonematic Systems
A. $\mathrm{C}_{1} \mathrm{~V}_{1}$
B. $\mathrm{C}_{2} \mathrm{~V}_{2}$

I. Prosodic Systems

A. Medial and Final Syllables $\left(C_{1} V_{1}+C_{2} V_{2}\right)$

A two-term prosodic system: $y / w$, hence ' $w$ Medial and Final' (wMF), and ' $y$ Medial and Final' (yMF). The exponents of $y / w$ need a statement in terms of $\mathrm{c} / \mathrm{o}$. In $\mathrm{cW}$ they may be stated only for $ə \mathrm{~W}$, not for $\mathrm{wW}$ and $\mathrm{yW}$. These exponents are :-

$(\mathrm{c} \partial \mathrm{W}) \quad-\mathrm{V}_{1}$

$\mathrm{w}:$ closeness + frontness dental plosion rounding

y: openness + centralization alveolar friction spreading, e.g. nasality

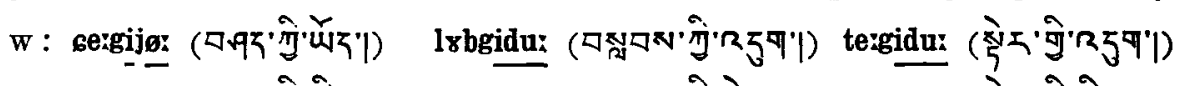

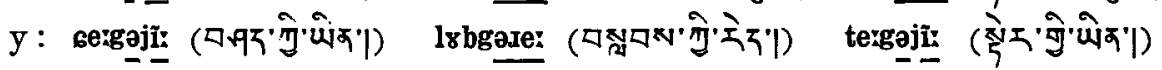

In oW exponents of $y / w$ may be stated only where the prosody of the medial syllable is 1 ( $\$ \mathrm{~B} 1$ below, $\left.1 \mathrm{M} /{ }^{\mathrm{n}} 1 \mathrm{M}\right)$, e.g. :-
(oW/1M)
$-\mathrm{V}_{1}$
$\mathrm{w}: \quad$ openness + frontness
$\mathrm{C}_{2^{-}}$
$-\mathrm{V}_{2}$
dental nasality rounding
+ plosion

y: closeness + centralization alveolar friction spreading \pm nasality, e.g.

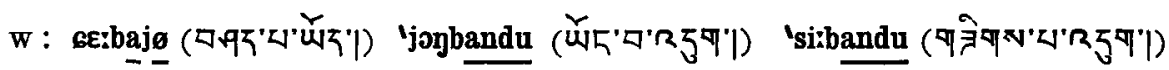

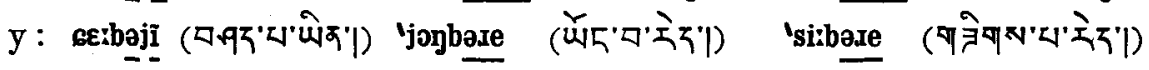

\section{B. (1) Medial Syllable $\left(C_{1} V_{1}\right)$}

Labialization: a two-term system $1 / n$, hence 'labial Medial (lM)', and ' non-labial Medial' ( $(\mathrm{n} I M)$. Exponents of this system may be stated only for the prosodic context yMF ( $\$$ A above). The phonetic exponents of these terms are :-

l: (1) $\mathrm{C}_{1^{-}}$: labial articulation

(2) $V_{1^{-}}$: half-closeness + central quality

$\mathrm{nl}_{\mathrm{l}}: \quad \mathrm{V}_{1^{-}}:$openness + frontness, e.g. :-

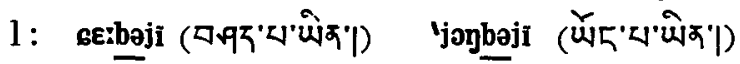

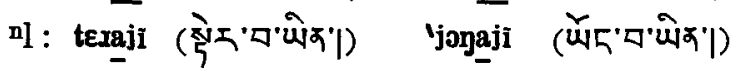

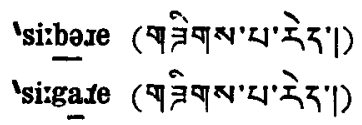

VOL. XVI. PART 3. 


\section{B. (2) Final Syllable $\left(\mathrm{C}_{2} \mathrm{~V}_{2}\right)$}

For the final syllable a prosodic system of closure may be stated: 'close' and 'open' (c/o), hence 'close Final' (cF) and 'open Final' (oF). The exponents of this system are :-

$$
\begin{aligned}
& C_{2^{-}} \\
& -\mathrm{V}_{2}
\end{aligned}
$$

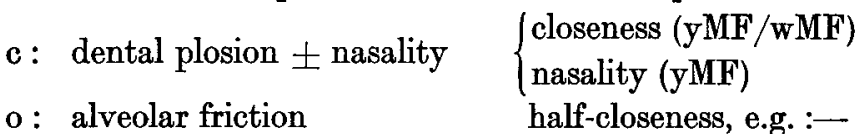

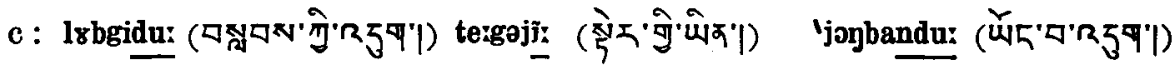

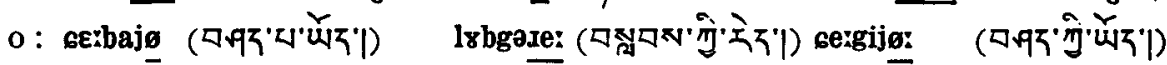

\section{Phonematic Systems}

A. Medial Syllable $\left(C_{1} V_{1}\right)$

The phonematic structure of the medial syllable comprises the following single terms in various prosodic contexts :-

(1) $-V_{1}$ :
(1) $-V_{1}$
(2) $\mathrm{C}_{1}-$

For the phonetic exponents of $-V_{1}$ the following prosodic systems must be taken into account :-
(a) $\mathrm{y} / \mathrm{w} / \mathrm{o} \quad$ (Word--W)
(b) $\mathrm{c} / \mathrm{o} \quad(,$,
(c) $y / w \quad$ (Medial and Final Syllables-MF)
(d) $1 / \mathrm{n}_{\mathrm{l}} \quad$ (Medial Syllable-M)

\begin{tabular}{|c|c|c|c|c|}
\hline $\mathrm{cW} / \mathrm{wW}:$ & 'drugydu: & 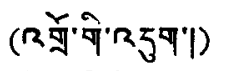 & 'jujgydu: & (स्पेन वें \\
\hline,$\quad \mathrm{yW}$ : & singijø: & (คेฟ.गु'น̌z'|) & tychigidu: & (1) \\
\hline əW/yMF : & 'deagejĩ: & 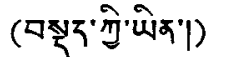 & tg6a:gaje: & \\
\hline , , wMF : & 'dergijø: & 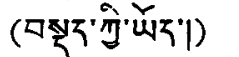 & ţ6s:gidu: & पip) \\
\hline $\mathrm{N} / \mathbf{n} \mathbf{M} \mathbf{M}$ & 'si: & 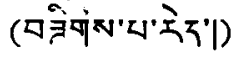 & nay & $5^{\circ} 1$ \\
\hline $\mathrm{lM} / \mathrm{yMF}:$ & $C E$ & Di) & 'jəgbəze & \\
\hline , wMF : & & $5 \%$ & 'jogbandu & ' \\
\hline
\end{tabular}

$-\mathrm{V}_{1}$ :

(2) $\mathrm{C}_{\mathrm{1}^{-}}$:

For the phonetic exponents of $\mathrm{C}_{1}$ - the following prosodic systems, together with the $-\mathrm{C}$ phonematic terms, must be taken into account :-

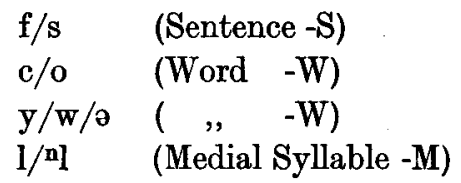

and the $-\mathrm{C}$ systems, e.g. :- 
$\mathrm{C}_{1^{-}}$:

$\mathrm{cW} / \mathrm{wW} /(-\mathrm{T} /-\mathrm{L} /-\mathrm{K}) / \mathrm{fS}$ :

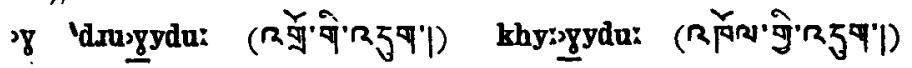
$\mathrm{BS}$ :

sg 'drusgydu: ( " ) khyıggydu: ( ", )

$\eta \quad, \quad-\mathbf{P} / \mathbf{f S}$ :

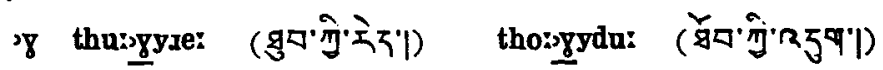
", , , sS :

'g thubsgyse: ( " ) thubrgydu: ( , ) $, \quad, \quad(-\mathrm{M} /-\mathrm{N} /-\mathrm{D})$ :

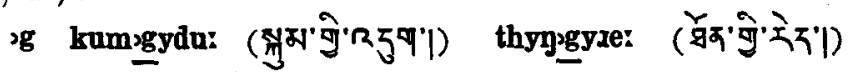
, $\quad \mathrm{yW} /(-\mathrm{T} /-\mathrm{L} /-\mathrm{K}) / \mathrm{fS}$ :

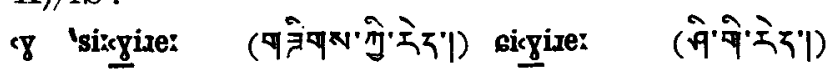

$$
\begin{aligned}
& \text {, , } \quad \text { SS : } \\
& \text { eg 'sikgire: ( ", ) eicgixe: ( ") ) } \\
& \text {, , }(-\mathrm{P} /-\mathrm{M} /-\mathrm{N} /-\mathrm{D}) \text { : }
\end{aligned}
$$

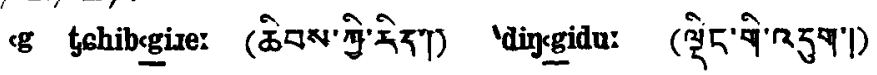
" $ә \mathrm{~W} /(-\mathrm{T} /-\mathrm{L} /-\mathrm{K}) \mathrm{fS}$ :

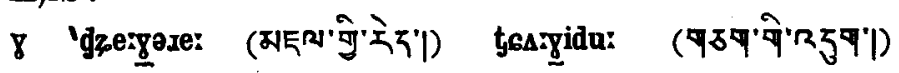

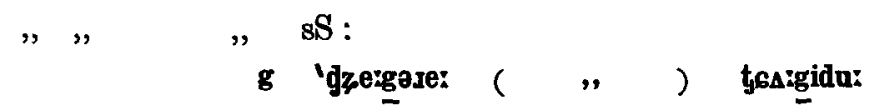$$
, \quad, \quad(-\mathrm{P} /-\mathrm{M} /-\mathrm{N} /-\mathrm{D}) \text { : }
$$

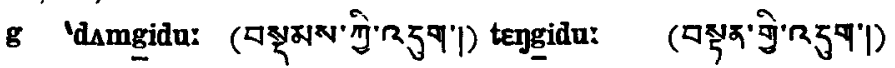
$\mathrm{oW} / 1 \mathrm{M} /(-\mathrm{T} / \mathrm{L} / \mathrm{-P}) \mathrm{fS}$ :

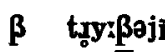

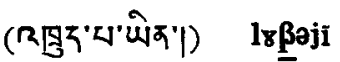

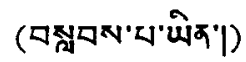

,$"$,

, $\mathrm{sS}$ :

b to̊y:beji

$, \quad, \quad(-\mathrm{K} /-\mathrm{M} /-\mathrm{N} /-\mathrm{D})$ :

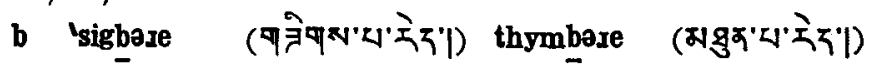

, $\mathrm{n} 1 \mathrm{M}$ :

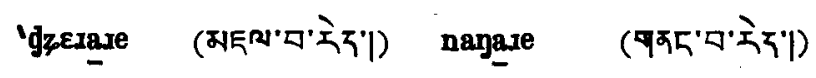

B. Final Syllable $\left(\mathrm{C}_{2} \mathrm{~V}_{2}\right)$

The phonematic structure of the final syllable comprise the following single terms in various prosodic contexts: (1) $-\mathrm{V}_{2},(2) \mathrm{C}_{2}-$. 
(1) $-V_{2}$

For the exponents of $-\mathrm{V}_{2}$ the following prosodic systems must be taken into account :-

(1) $1 / 2$ (Sentence-S ; $(a)$ of one word only ; $(b)$ of more than one word, pp. 141-6 above).

(2) c/o (Word-W)

(3) $y / w$ (Medial and Final Syllables-MF)

(4) c/o (Final Syllable-F), e.g. :$-\mathrm{V}_{2}$ $1 \mathrm{~S}(\mathrm{a}) / \mathrm{cW} / \mathrm{wMF} / \mathrm{cF}$ :

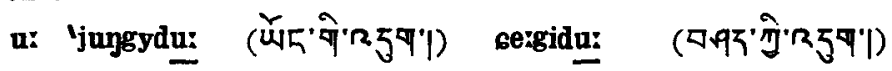

,(b) , , , , :

u:: 'juggydu:: ( , ) ce:gidu: ( , ) 2S(a) , , , , :

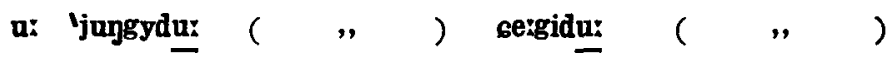
, (b) , , , :

u 'jungydu $("$,$) seigidu (")$ oW , , :

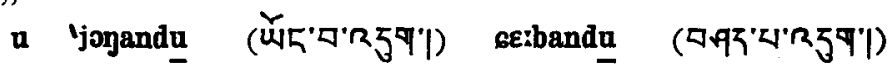
$1 \mathrm{~S}(\mathrm{a}) / \mathrm{cW} / \mathrm{wMF} / \mathrm{oF}$ :

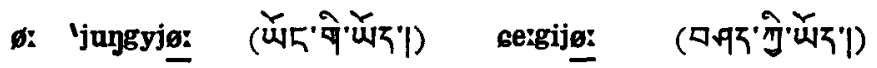
,(b) , , , :

6i: 'jungyjø:: ( $")$ gezgijg:: (,$\quad)$ $2 \mathrm{~S}(\mathrm{a}) \quad, \quad, \quad, \quad$ :

ø: 'juggyjø: ( " ) cegijø: ( " ) ,(b) , , , , :

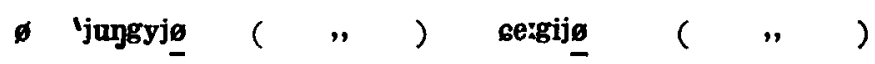
oW/ , , : :

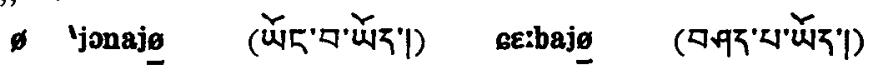
$1 \mathrm{~S}(\mathrm{a}) / \mathrm{cW} / \mathrm{yMF} / \mathrm{cF}$ :

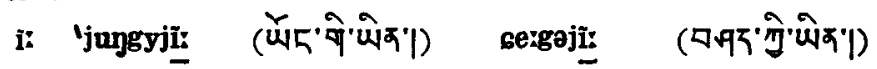
, (b) , , , , :

in: 'juggyili: ( $"$ ) ceagojĩ: $("$, $2 \mathrm{~S}(\mathrm{a}) \quad, \quad, \quad, \quad$ :

i: 'juggyjĩ: ( 
$2 \mathrm{~S}(\mathrm{~b}) / \mathrm{cW} / \mathrm{yMF} / \mathrm{cF}$ :

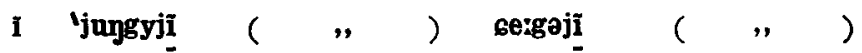

$$
\begin{aligned}
& \text { oW/ , , : }
\end{aligned}
$$

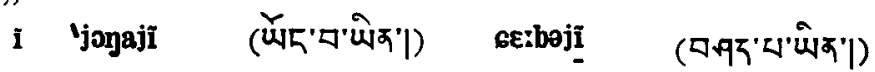

$1 \mathrm{~S}(\mathrm{a}) / \mathrm{cW} / \mathrm{yMF} / \mathrm{oF}$ :

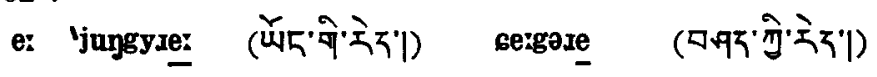

, (b) , , , , :

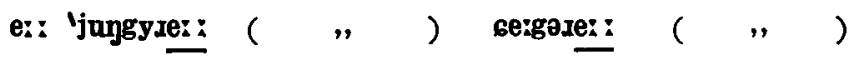

2S(a) ,, , ,, :

e: 'jūggyze: ( , ) cergəze: ( , )

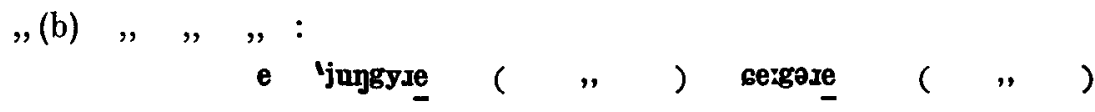

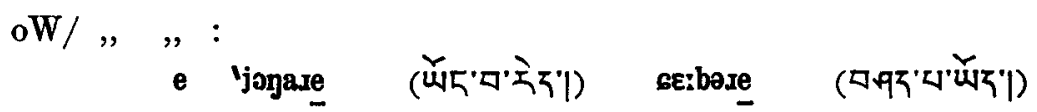

(2) $\mathrm{C}_{\mathbf{2}^{-}}$,

For the exponents of $\mathrm{C}_{2}$ - the following prosodic systems have to be taken into account :-

$$
\begin{array}{ll}
\text { f/s } & \text { (Sentence-S) } \\
\text { c/o } & (\text { Word }-W) \\
\text { w/n } & (\text { " }-n) \\
\text { y/w } & \text { (Medial and Final Syllables-MF) } \\
\text { c/o } & \text { (Final Syllable-F), e.g. :- }
\end{array}
$$

$\mathrm{fS} / \mathrm{cW} / \mathrm{wMF} / \mathrm{cE}$ :

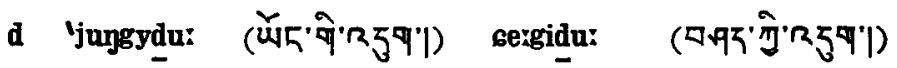
sS/ , , , :

nd ljuggynda: ( , ) seigindu: ( " ) oW/ , , , :

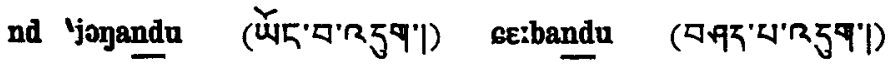
$\mathrm{wW} / \mathrm{cW} /, \quad / \mathrm{oF}:$

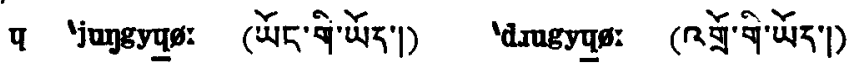
$\mathrm{n}_{\mathrm{WW}} \mathrm{W},, \quad, \quad, \quad$ :

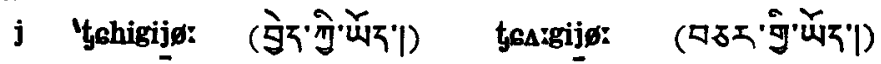
$\mathbf{o W} /,,$,

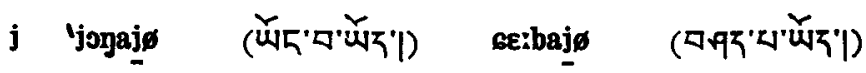




$$
\begin{aligned}
& \mathrm{yMF} / \mathrm{cF} \text { : }
\end{aligned}
$$

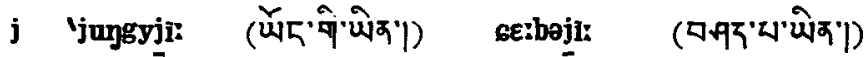

$$
\begin{aligned}
& \mathrm{wW} / \mathrm{cW} /,, / \mathrm{oF} \text { : }
\end{aligned}
$$

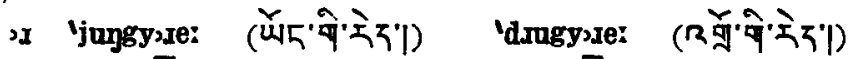

$$
\begin{aligned}
& \mathrm{n}_{\mathrm{wW}} \mathrm{W},, \quad, \quad, \quad \text { : }
\end{aligned}
$$

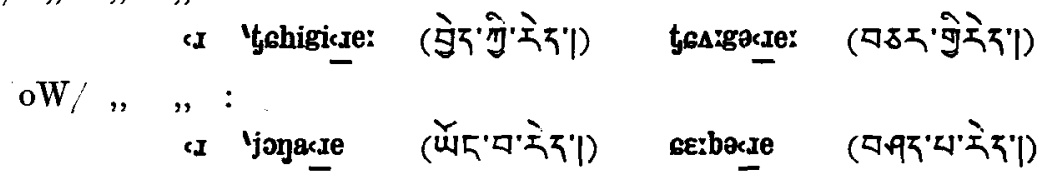

The prosodic and phonematic statements made above are limited to the trisyllabic verbal phrase. If that were to be extended by the addition of par-

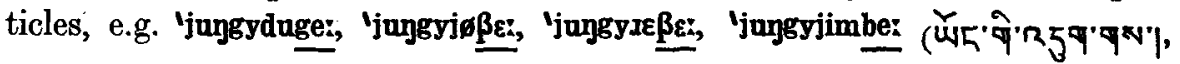

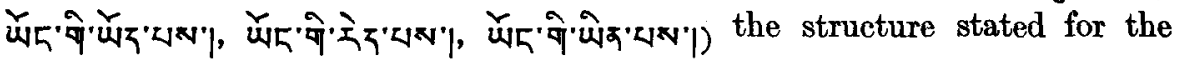
Particle 2 might need to be reconsidered.

A point that seems clearly to emerge from the prosodic and phonematic statements made for the Final Syllable (Particle 2) is that no useful purpose is

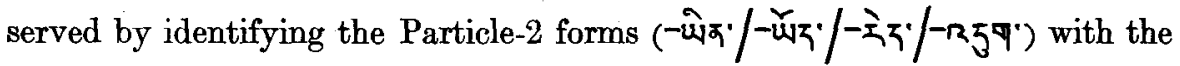

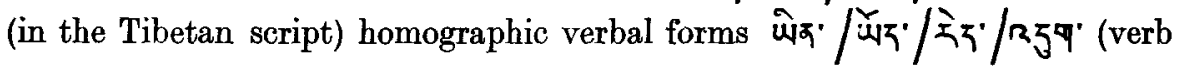
complement). The prosodic statements made for the particle would not be valid for the verb complement. Still more unsatisfactory, and for the same reason, would be any attempt to identify the Particle 1 (पें/ पें / गें and $\left.\square^{\prime} / \mathbf{J}^{\prime} / \Sigma^{\prime} / \nabla^{\prime} / \Sigma^{\prime}\right)$ of the trisyllabic verbal phrase with homographic noun particles वें / शें / गें' and $\square^{\circ} / \mathrm{U}^{\circ}$, for it is such an identification as this that is the basis of the references:-

'The Tibeto-Burman verb is properly a noun,' ' . . . the nominal character of the Tibetan verb...'1. Such statements cannot stand for contemporary LT, if phonological and grammatical statements are to be congruent.

This congruence of statement, which has been the aim of the phonological analysis of the material presented above, is illustrated in the following section.

\section{PROSODIC SYSTEM OF THE SYLLABLE FINAL, TENSE, AND ASPECT}

A prosodic system of the syllable final $(y / w F)$ has been stated for the initial syllable of $\mathrm{wW}$ and $\mathrm{yW}$, and is implicit in the stating of $ə \mathrm{~W}$ in terms of the prosodic system of $y / w$ for the initial syllable as a whole. It is this system

1 Linguistic Survey of India, vol. iii, pt. i, Calcutta, 1909, pp. 8 and 9 respectively. cf. also Hannah: 'As a matter of fact the only real verb in Tibetan is the verb To be, whether in the form

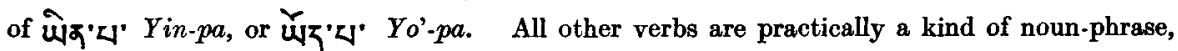
dependent for their significations upon the various moods and tenses of these two verbs শিহ ‘'

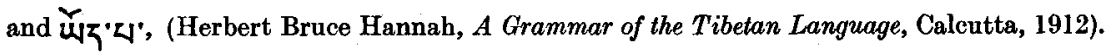


which was referred to in Part I under the term flexion as one of the criteria for defining the verb. The system was particularly associated with the $-\mathrm{C}$ term -T. For many, but not all, syllables in $-T$, all of them being at the grammatical level of analysis verbs, it would be possible to state the $w$ and $y$ terms of the $\mathrm{F}$ system as phonological exponents, among other exponents, of tense (present and past respectively). ${ }^{1}$ Examples of these verbs in - $T$ are as follows :-

$-\mathbf{T}$

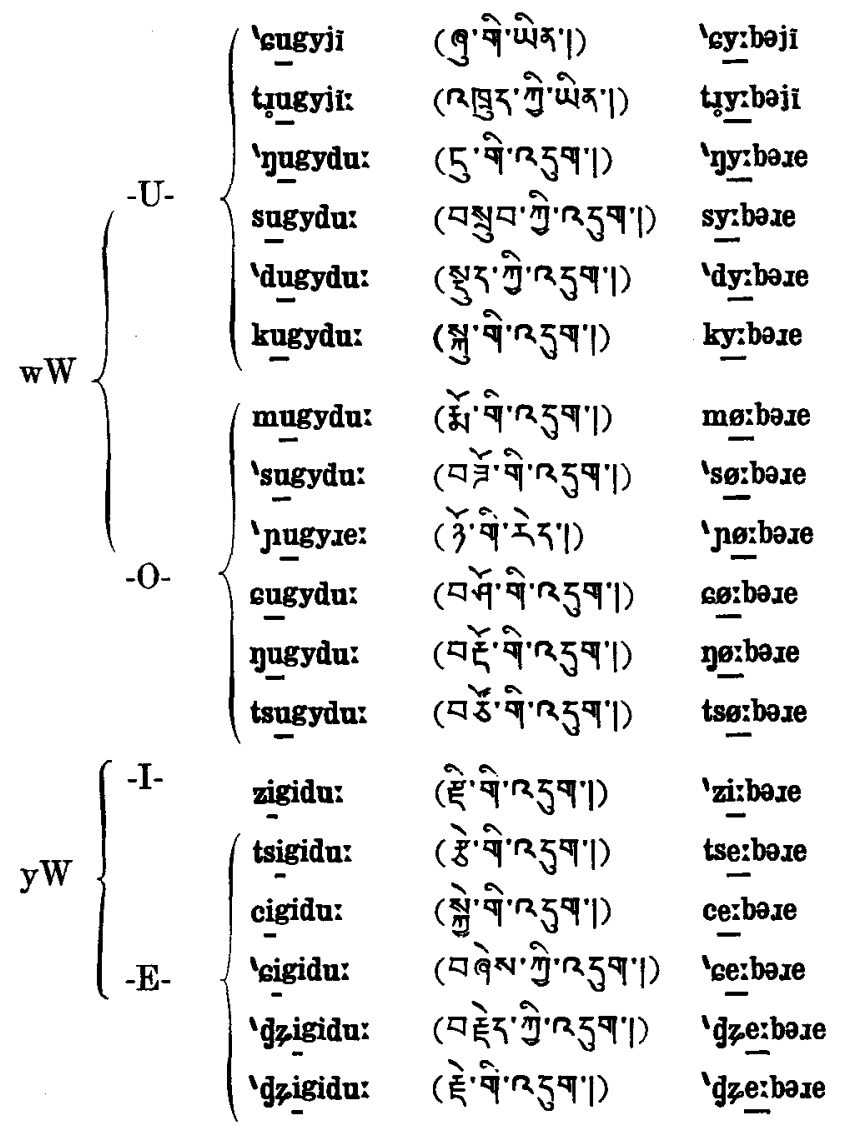

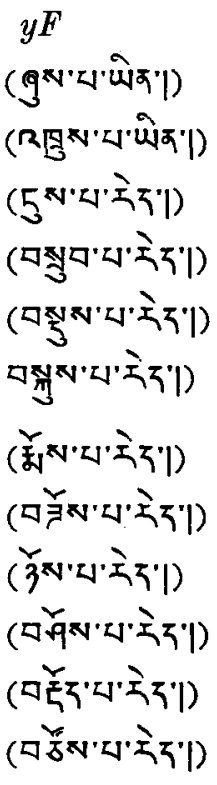

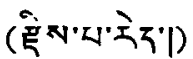

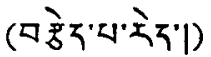

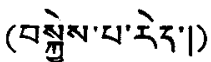

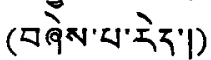

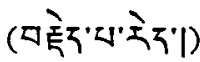

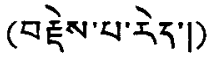

1 Having committed myself in Part II to the stating of certain phonetic features as exponents of a $-\mathrm{C}$ term - $T$, with the implication that for syllables in - $T$ a prosodic system of the syllable final $(y / w)$ may be stated in $y W$ and $w W$, and a $y / w$ prosodic system of the syllable for $ə W, I$ am unable at this late stage to reconsider and restate this material. I should now prefer, however, to restate as syllables of a CV structure those syllables which I bave hitherto stated as syllables in the $-\mathrm{C}$ term - $T$, and to retain as syllables of a CVC structure syllables hitherto stated as syllables in -C term other than -T (i.e. -L/-P/-M, etc.). The grounds for this change in statement are that while it is advantageous to state a $y / w F$ system for syllables treated above as syllables in - $T$ in order to secure congruence with a particular grammatical statement no such consideration applies to syllables in -C terms other than those in - $T$. In my grammatical statement $I$ shall need to distinguish two categories of verb; the possibility of stating a CV structure as a phonological exponent of one category and a CVC structure as an ex onent of the other should not be left unexploited. 
$-\mathbf{T}$

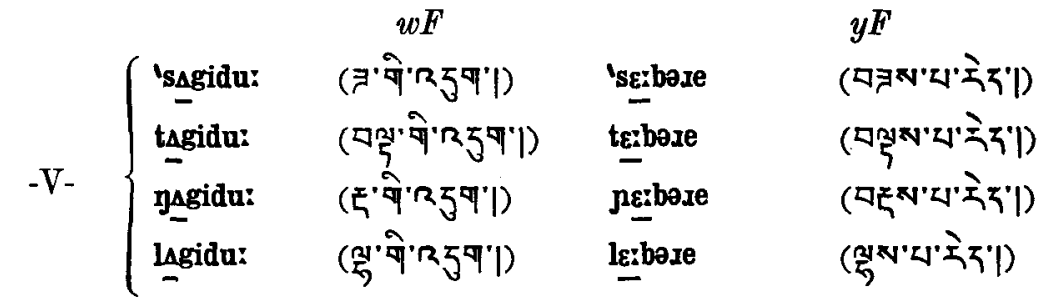

Among the verbs in - $\mathrm{T}$ for which $\mathrm{wF}$ and $\mathrm{yF}$ prosodies may not be stated as phonological exponents of tense categories are the following; for them only ${ }_{w} \mathrm{~F}$ is statable :-

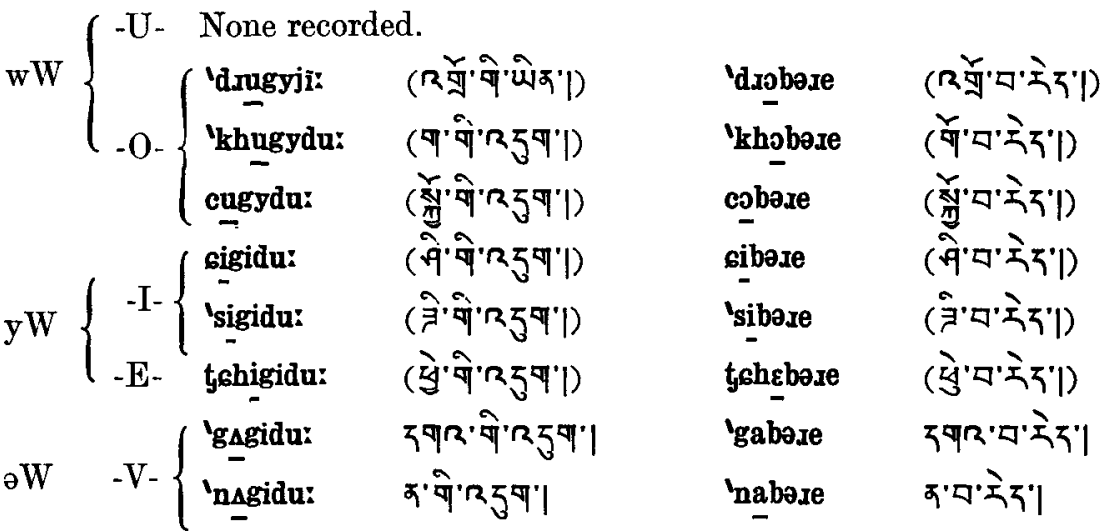

For one verb in $-\mathrm{T}$ both $\mathrm{wF}$ and $\mathrm{yF}$ prosodies have been recorded, but together with $\mathrm{yW}$ in one prosodic context (cW) and with $ə \mathrm{~W}$ in the other (oW) ; all other examples of verbs in - $\mathrm{T}$ have a common prosodic feature, whether $\mathrm{w}, \mathrm{y}$, or $\partial$, in $\mathrm{oW}$ and $\mathrm{cW}$ alike :-$$
\mathrm{yW}, \underline{\mathrm{wF}}
$$

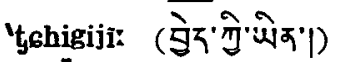

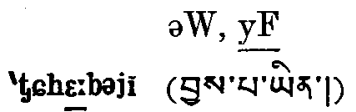

In the above examples the wF forms have been confined to $\mathrm{cW}$ and the $\mathrm{y} F$ to oW. Examples have, however, been recorded where both $\mathrm{yF}$ and $\mathrm{wF}$ might be stated for oW, e.g.

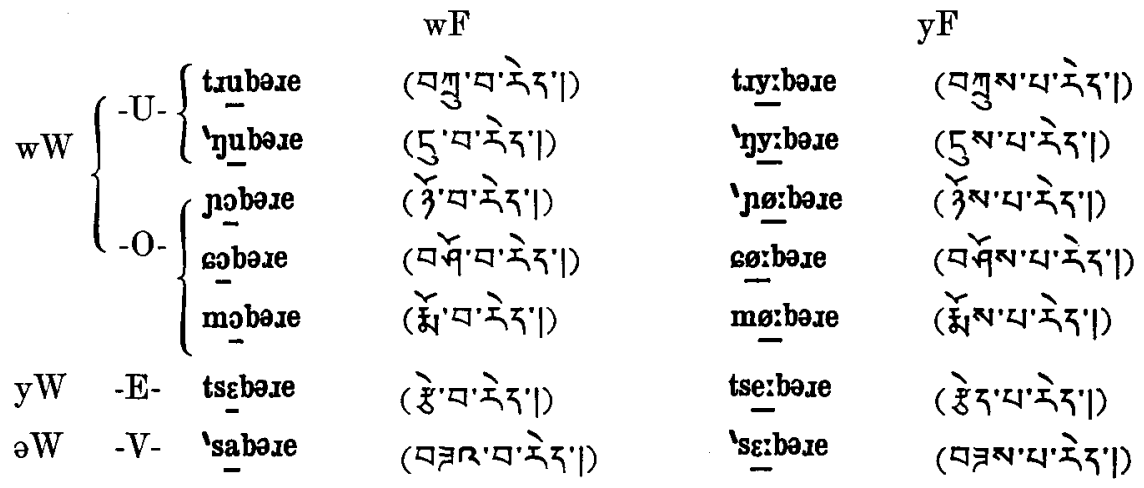


Where both terms of the prosodic system of the syllable final may be stated for $\mathrm{oW}$ for a given verb the two terms may be treated as phonological exponents of a grammatical category of aspect. Thus $\mathrm{wF}$ might be stated as an exponent of ' habitual' and yF of 'incidental' aspect,' e.g.

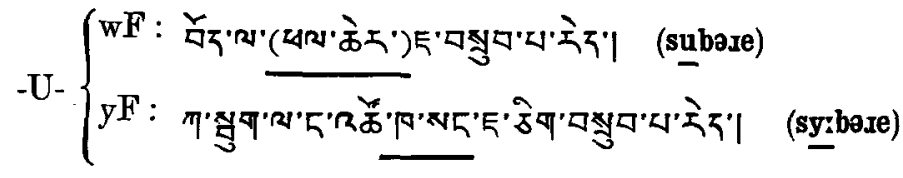

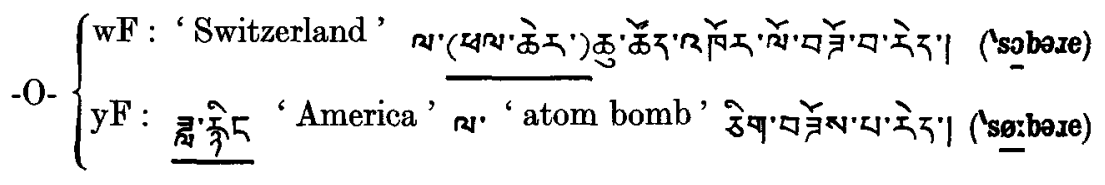

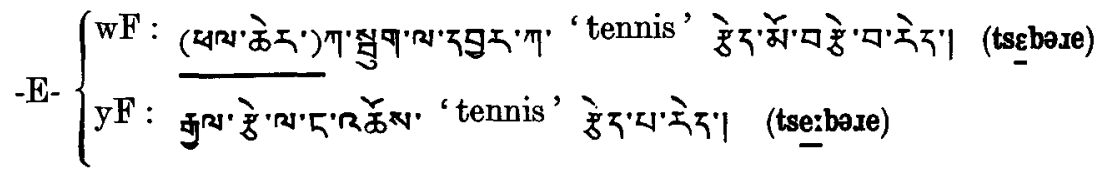

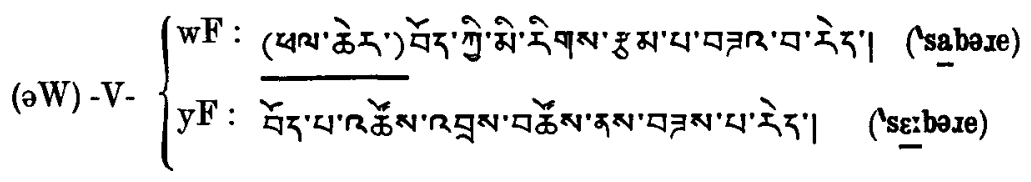

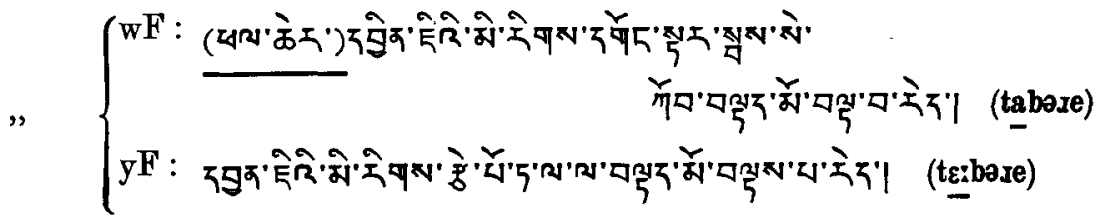

Statements such as those above cannot, however, be made for all verbs in $-\mathrm{T}$; certain verbs have only one form, either $\mathrm{yF}$ or $\mathrm{wF}$, in the same prosodic context (oW), e.g.

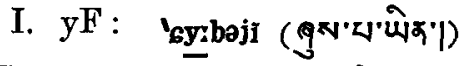

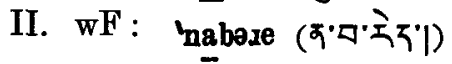

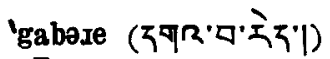

1 The two terms referred to here as 'habitual' and 'incidental' do not constitute a closed system of aspect; such a system would comprise more than these two terms, with exponents, both in Verbal and Nominal Phrase, different from those exemplified here, and beyond the scope of

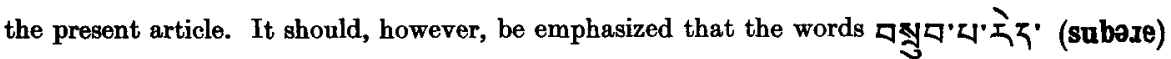

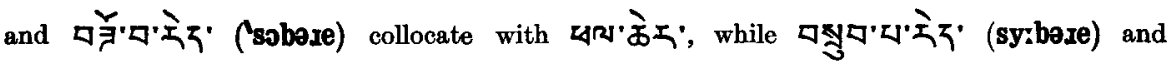

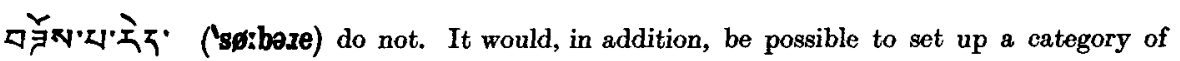

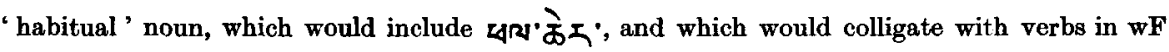
and not with verbs in $\mathrm{yF}$. A similarly established category of 'incidental' noun, including $\beta^{\prime} \times 5^{\prime}$, would colligate with verbs in $\mathrm{yF}$. 